\title{
Generating unconstrained two-dimensional non-guillotine cutting patterns by a recursive partitioning algorithm
}

\author{
Ernesto G. Birgin * Rafael D. Lobato * Reinaldo Morabito ${ }^{\dagger}$
}

April 3, 2010

\begin{abstract}
In this study, a dynamic programming approach to deal with the unconstrained twodimensional non-guillotine cutting problem is presented. The method extends the recently introduced recursive partitioning approach for the manufacturer's pallet loading problem. The approach involves two phases and uses bounds based on unconstrained two-staged and non-staged guillotine cutting. The method is able to find the optimal cutting pattern of a large number of problem instances of moderate sizes known in the literature and a counterexample for which the approach fails to find known optimal solutions was not found. For the instances that the required computer runtime is excessive, the approach is combined with simple heuristics to reduce its running time. Detailed numerical experiments show the reliability of the method.
\end{abstract}

Key words: Cutting and packing, two-dimensional non-guillotine cutting pattern, dynamic programming, recursive approach, distributor's pallet loading problem.

\section{Introduction}

In the present paper, we study the generation of two-dimensional non-guillotine cutting (or packing) patterns, also referred by some authors as two-dimensional knapsack problem or two-dimensional distributor's pallet loading. This problem is classified as $2 / \mathrm{B} / \mathrm{O} /$ according to Dyckhoff's typology of cutting and packing problems [21], and as two-dimensional rectangular Single Large Object Packing Problem (SLOPP) based on Waescher et al.'s typology [47]. Besides the inherent complexity of this problem (it is NP-hard [7]), we are also motivated by its practical relevance in different industrial and logistics settings, such as in the cutting of steel and glass stock plates into required sizes, the cutting of wood sheets and textile materials to make ordered pieces, the loading of different items on the pallet surface or the loading of different pallets on the truck or container floor, the cutting of cardboards into boxes, the placing of advertisements on

\footnotetext{
${ }^{*}$ Department of Computer Science, Institute of Mathematics and Statistics, University of São Paulo, Rua do Matão 1010, Cidade Universitária, 05508-090 São Paulo, SP - Brazil. e-mail: \{egbirgin|lobato\}@ime.usp.br

${ }^{\dagger}$ Department of Production Engineering, Federal University of São Carlos, Via Washington Luiz km. 235, 13565-905, São Carlos, SP - Brazil. e-mail: morabito@ufscar.br

${ }^{\ddagger}$ Revised on August 20, 2010.
} 
the pages of newspapers and magazines, the positioning of components on chips when designing integrated circuit, among others.

Given a large rectangle of length $L$ and width $W$ (i.e. a stock plate), and a set of rectangular pieces grouped into $m$ different types of length $l_{i}$, width $w_{i}$ and value $v_{i}, i=1, \ldots, m$ (i.e. the ordered items), the problem is to find a cutting (packing) pattern which maximizes the sum of the values of the pieces cut (packed). The cutting pattern is referred as two-dimensional since it involves two relevant dimensions, the lengths and widths of the plate and pieces. A feasible two-dimensional pattern for the problem is one in which the pieces placed into the plate do not overlap each other, they have to be entirely inside the plate, and each piece must have one edge parallel to one edge of the plate (i.e., an orthogonal pattern). In this paper we assume that there are no imposed lower or upper bounds on the number of times that each type of piece can be cut from the plate; therefore, the two-dimensional pattern is called unconstrained (i.e., 0 and $\left\lfloor\frac{L}{l_{i}}\right\rfloor\left\lfloor\frac{W}{w_{i}}\right\rfloor$ turn out to be the obvious minimum and maximum number of pieces of type $i$ in the pattern); otherwise, it would be called constrained or doubly-constrained.

Without loss of generality, we also assume that the cuts are infinitely thin (otherwise we consider that the saw thickness was added to $\left.L, W, l_{i}, w_{i}\right)$, the orientation of the pieces is fixed (i.e., a piece of size $\left(l_{i}, w_{i}\right)$ is different from a piece of size $\left(w_{i}, l_{i}\right)$ if $\left.l_{i} \neq w_{i}\right)$ and that $L, W$, $l_{i}, w_{i}$ are positive integers. We note that if the $90^{\circ}$-rotation is allowed for cutting or packing the piece type $i$ of size $\left(l_{i}, w_{i}\right)$, this situation can be handled by simply considering a fictitious piece type $m+i$ of size $\left(w_{i}, l_{i}\right)$ in the list of ordered items, since the pattern is unconstrained. Depending on the values $v_{i}$, the pattern is called unweighted, if $v_{i}=\gamma l_{i} w_{i}$ for $i=1, \ldots, m$ and $\gamma>0$ (i.e., proportional to the area of the piece), or weighted, otherwise.

Moreover, we assume that the unconstrained two-dimensional cutting pattern is non-guillotine as it is not limited by the guillotine type cuts imposed by some cutting machines (an orthogonal guillotine cut on a rectangle is a cut from one edge of the rectangle to the opposite edge, parallel to the remaining edge). Some industrial cutting processes also limit the way of producing a guillotine cutting pattern. At a first stage the cuts are performed parallel to one side of the plate and then, at the next stage, orthogonal to the preceding cuts, and so on. If there is an imposed limit on the number of stages, say $k$, the guillotine pattern is called a $k$-staged pattern; otherwise, it is non-staged (note that a non-staged pattern is equivalent to define $k$ large enough).

A large number of studies in the literature have considered staged and non-staged twodimensional guillotine cutting problems. Much less studies have considered two-dimensional non-guillotine cutting problems (constrained and unconstrained), and only a few of them have proposed exact methods to generate non-guillotine patterns. For example, [6] and [28] presented 0-1 linear programming formulations for the problem using $0-1$ decision variables for the positions of the pieces cut from the plate; they developed Lagrangean relaxations and used them as bounds in tree search procedures (subgradient optimization was used to optimize the bounds). In [46] and [16], the problem is formulated as $0-1$ linear models using left, right, top and bottom decision variables for the relative positions of each pair of pieces cut from the plate (with multiple choice disjunctive constraints); they suggested solving the models by branch-and-bound algorithms exploring particular structures of these constraints. Other related $0-1$ linear formulations appear in [14, 22], and a 0-1 non-linear formulation was presented in [7] (see also [44, 42]). Other exact and branch-and-bound approaches based on the so called two-level approach (the 
first level selects the set of pieces to be cut without taking into account the layout, the second checks whether a feasible cutting layout exists for the pieces selected) are found in [15, 4, 24]. The method presented in [24] is based on a two-level tree search that combines the use of a special data structure for characterizing feasible cuttings with upper bounds.

Different heuristics based on random local search, bottom-left placement, network flow, graph search, etc., and different metaheuristics based on genetic algorithms, tabu search, simulated annealing, GRASP, etc., for generating constrained and unconstrained two-dimensional nonguillotine cuttings are found in the literature. Some recent examples are in $[2,27,35,17,13$, $22,48]$. Nonlinear-programming-based method for packing rectangles within arbitrary convex regions, considering different types of positioning constraints, were presented in [10, 11, 8, 38].

Most of these approaches were developed for the constrained problem, which can be more interesting for certain practical applications with relatively low demands of the ordered items. However, part of these methods may not perform well when solving the unconstrained problem, especially those whose computational performance is highly dependent on the total number of ordered items. On the other hand, the unconstrained problem is particularly interesting for cutting stock applications with large-scale production and weakly heterogeneous items (i.e., relatively few piece types but many copies per type), in which the problem plays the role of a column generation procedure, as pointed out by several authors since the pioneer study in [26].

In the present paper we extend a Recursive Partitioning Approach presented in [9] for the manufacturer's pallet loading to deal with the unconstrained two-dimensional orthogonal nonguillotine cutting (unweighted and weighted, without and with piece rotation). This Recursive Partitioning Approach combines refined versions of both the Recursive Five-block Heuristic presented in $[40,41]$ and the $L$-approach for cutting rectangles from larger rectangles and $L$-shaped pieces presented in $[37,12])$. This combined approach also uses bounds based on unconstrained two-staged and non-staged guillotine cutting patterns. The approach was able to find an optimal solution of a large number of problem instances of moderate sizes known in the literature and we were unable to find an instance for which the approach fails to find a known or proved optimal solution. For the instances that the required computer runtimes were excessive, we combined the approach with simple heuristics to reduce its running time.

The paper is organized as follows. In Section 2 we briefly review a $0-1$ linear programming formulation of the problem and some lower and upper bounds known in the literature. In Section 3 we present the two phases of the Recursive Partitioning Approach and some heuristics that reduce the time and memory requirements of the procedure to deal with large problem instances. Then in Section 4 we analyze the computational performance of the approach, with and without the heuristics, in some numerical experiments. Finally, in Section 5 we present the concluding remarks of this study.

\section{Problem modeling and bounds}

\subsection{Problem modeling}

The unconstrained two-dimensional non-guillotine cutting problem can be modeled as a $0-1$ linear formulation proposed in [6]. Let $(x, y)$ be the coordinates of the left-lower-corner of a piece placed on the plate of size $(L, W)$ (for simplicity we assume that the left-lower-corner of 
the plate is $(0,0))$. Without loss of generality, it can be shown that $x$ and $y$ belong, respectively, to the sets of conic combinations of $l_{i}$ and $w_{i}, i=1, \ldots, m$, for $L$ and $W$, defined by:

$$
\begin{aligned}
S_{L} & =\left\{x \in \mathbb{Z}_{+} \mid x=\sum_{i=1}^{m} r_{i} l_{i}, 0 \leq x \leq L, r_{i} \in \mathbb{Z}_{+}, i=1, \ldots, m\right\} \text { and } \\
S_{W} & =\left\{y \in \mathbb{Z}_{+} \mid y=\sum_{i=1}^{m} s_{i} w_{i}, 0 \leq y \leq W, s_{i} \in \mathbb{Z}_{+}, i=1, \ldots, m\right\} .
\end{aligned}
$$

Note that as we decide to place a piece of type $i$ in a position $(x, y)$, we cannot place another piece in a position $\left(x^{\prime}, y^{\prime}\right)$ such that $x \leq x^{\prime} \leq x+l_{i}-1$ and $y \leq y^{\prime} \leq y+w_{i}-1, x, x^{\prime} \in S_{L}$, $y, y^{\prime} \in S_{W}$. In order to avoid piece overlapping, let $g_{i x y x^{\prime} y^{\prime}}$ be the mapping:

$$
g_{i x y x^{\prime} y^{\prime}}= \begin{cases}1, & \text { if } x \leq x^{\prime} \leq x+l_{i}-1 \text { and } y \leq y^{\prime} \leq y+w_{i}-1 \\ 0, & \text { otherwise }\end{cases}
$$

which can be computed a priori for each $i,(x, y)$ and $\left(x^{\prime}, y^{\prime}\right)$. By defining the decision variables $a_{i x y}, i=1, \ldots, m, x \in S_{L}, y \in S_{W}$, as:

$$
a_{i x y}= \begin{cases}1, & \text { if a piece of type } i \text { is placed in a position }(x, y), \\ 0, & \text { otherwise, }\end{cases}
$$

the model can be formulated as the following 0-1 integer program:

$$
\begin{aligned}
\max & \sum_{i=1}^{m} \sum_{x \in S_{L}} \sum_{y \in S_{W}} v_{i} a_{i x y} \\
\text { subject to } & \sum_{i=1}^{m} \sum_{x \in S_{L}} \sum_{y \in S_{W}} g_{i x y x^{\prime} y^{\prime}} a_{i x y} \leq 1, \quad x^{\prime} \in S_{L}, y^{\prime} \in S_{W}, \\
& a_{i x y} \in\{0,1\}, i=1, \ldots, m, x \in S_{L}, y \in S_{W} .
\end{aligned}
$$

Note that model (1-3) has $O\left(m\left|S_{L}\right|\left|S_{W}\right|\right) 0-1$ variables and $\left|S_{L}\right|\left|S_{W}\right|$ constraints $^{1}$. It can be shown that, without loss of generality, sets $S_{L}$ and $S_{W}$ can be replaced by the smaller sets $R_{L}$ and $R_{W}$ known as reduced raster points [45] and defined as:

$$
\begin{aligned}
R_{L} & =\left\{x \in \mathbb{Z}_{+} \mid x=\langle L-\hat{x}\rangle_{S_{L}} \text { for some } \hat{x} \in S_{L}\right\}, \\
R_{W} & =\left\{y \in \mathbb{Z}_{+} \mid y=\langle W-\hat{y}\rangle_{S_{W}} \text { for some } \hat{y} \in S_{W}\right\},
\end{aligned}
$$

where

$$
\langle\tilde{x}\rangle_{S_{L}}=\max \left\{x \in S_{L} \mid x \leq \tilde{x}\right\} \text { and }\langle\tilde{y}\rangle_{S_{W}}=\max \left\{y \in S_{W} \mid y \leq \tilde{y}\right\} .
$$

However, since both $S_{L}, S_{W}$ and $R_{L}, R_{W}$ can be large in practical cases, it may be hard to solve the model above, as illustrated in Section 4. As mentioned before, other 0-1 linear models for the non-guillotine cutting are found in the literature, but authors have pointed out that their LP-relaxations provide bounds in general far from the optimal solution values [7, 22].

\footnotetext{
${ }^{1}$ By defining $S_{L}^{i}=\left\{x \in S_{L} \mid 0 \leq x \leq L-l_{i}\right\}$ and $S_{W}^{i}=\left\{y \in S_{W} \mid 0 \leq y \leq W-w_{i}\right\}$, it is possible to reduce the number of binary variables of the model substituting $S_{L}$ and $S_{W}$ by $S_{L}^{i}$ and $S_{W}^{i}$, respectively, in the summations of (1) and (2) and in (3).
} 


\subsection{Lower and upper bounds}

A simple lower bound on the value of an optimal cutting can be obtained from the best homogeneous packing considering all types of pieces:

$$
\max _{i \in\{1, \ldots, m\}}\left\{v_{i}\left\lfloor\frac{L}{l_{i}}\right\rfloor\left\lfloor\frac{W}{w_{i}}\right\rfloor\right\} .
$$

Similarly, a simple upper bound on the value of an optimal cutting is given by

$$
\left\lfloor L W \max _{i \in F(L, W)}\left\{\frac{v_{i}}{l_{i} w_{i}}\right\}\right\rfloor,
$$

where $F(L, W)=\left\{i \in\{1, \ldots, m\} \mid l_{i} \leq L\right.$ and $\left.w_{i} \leq W\right\}$.

Other lower and upper bounds are found in $[6,43,25,7,2,4]$. However, most of these lower and upper bounds were proposed for the constrained non-guillotine cutting, and they are less effective for the unconstrained problem. In our implementation of the algorithm described in Section 3, we have used the lower bound given by the best homogeneous packing (5) and the upper bound introduced in [25]. According to [25], the upper bound for a rectangle with length $L$ and width $W$ considering all types of pieces is given by

$$
\min \left\{u_{h}(L, W), u_{v}(L, W)\right\},
$$

where

$$
u_{h}(L, W)=\left\lfloor W \max _{t \in \mathbb{Z}_{+}^{m}}\left\{\sum_{i \in F(L, W)} \frac{v_{i}}{w_{i}} t_{i} \mid \sum_{i \in F(L, W)} l_{i} t_{i} \leq L\right\}\right\rfloor
$$

and

$$
u_{v}(L, W)=\left\lfloor L \max _{t \in \mathbb{Z}_{+}^{m}}\left\{\sum_{i \in F(L, W)} \frac{v_{i}}{l_{i}} t_{i} \mid \sum_{i \in F(L, W)} w_{i} t_{i} \leq W\right\}\right\rfloor .
$$

Upper bounds for all subproblems can be obtained by solving only two unconstrained knapsack problems at the beginning of the algorithm. A dynamic programming procedure for solving these problems is presented in details in [25].

\section{Description of the algorithm}

The Recursive Partitioning Algorithm presented here is an extension of the algorithm described in [9] for the manufacturer's pallet loading problem. It has basically two phases: in phase 1 it applies a recursive five-block heuristic based on the procedure presented in [40] and in phase 2 it uses an $L$-approach based on a dynamic programming recursive formula presented in $[37,12]$. Firstly, phase 1 is executed and, if a certificate of optimality is not provided by the Recursive Five-block Heuristic, then phase 2 is executed. Additionally, information obtained in phase 1 is used in phase 2 in at least two ways, according to [9]. If an optimal solution was already found for a subproblem in phase 1 , it is not solved again in phase 2, improving the performance of phase 2. Moreover, having the information obtained in phase 1 at hand, phase 2 is often able to obtain better lower bounds for its subproblems than the ones provided by homogeneous cuttings (5), therefore improving the performance of phase 2 . These two phases are detailed in the sequel. 


\subsection{Phase 1}

In phase 1, the Recursive Five-block Heuristic divides a rectangle into five (or less) smaller rectangles in a way that is called first-order non-guillotine cut [3]. Figure 1 illustrates this kind of cut represented by a quadruple $\left(x_{1}, x_{2}, y_{1}, y_{2}\right)$, such that $0 \leq x_{1} \leq x_{2} \leq L$ and $0 \leq y_{1} \leq y_{2} \leq W$. This cut determines five subrectangles $\left(L_{1}, W_{1}\right), \ldots,\left(L_{5}, W_{5}\right)$ such that $L_{1}=x_{1}, W_{1}=W-y_{1}$, $L_{2}=L-x_{1}, W_{2}=W-y_{2}, L_{3}=x_{2}-x_{1}, W_{3}=y_{2}-y_{1}, L_{4}=x_{2}, W_{4}=y_{1}, L_{5}=L-x_{2}$ and $W_{5}=y_{2}$. Each rectangle is recursively cut unless the (sub)problem related to this rectangle has already been solved.

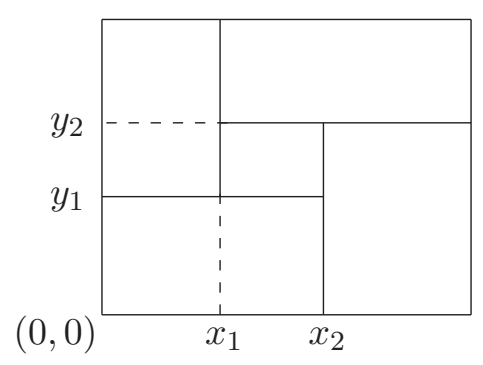

(a)

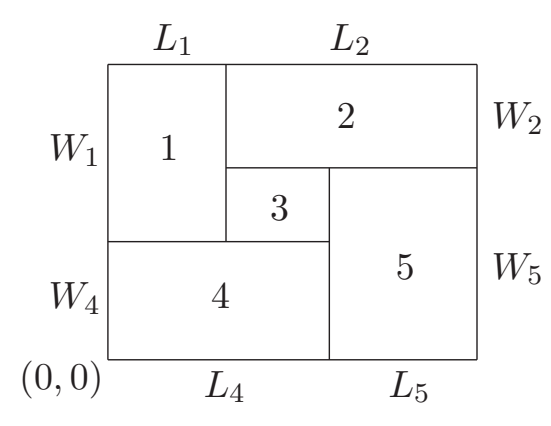

(b)

Figure 1: Representation of a first-order non-guillotine cut.

A pseudo-code of the Recursive Five-block Algorithm for the unconstrained non-guillotine cutting problem is presented in Algorithms $1^{2}$ and 2. The method is composed by two procedures: Five-Block-Algorithm and Solve. The second one is an auxiliary subroutine used by the first. Input parameters of Five-BLOCK-Algorithm include the dimensions of the plate and the pieces, and $n$ and $N$ correspond to the current depth and the maximum depth limit of the search, respectively.

In Five-BlOCK-Algorithm, the lower bound starts with the value of the best homogeneous solution (5), and it is updated as better solutions are found by the algorithm. The procedure uses the upper bound (6). Both the initial lower and upper bounds are computed a priori. In this way, an optimal solution can be detected in the procedure by closing the gap between these bounds. Note that without loss of generality, the procedure only considers the sets of raster points as defined in Section 2. Moreover, the same symmetry constraints for both guillotine and first-order non-guillotine cuttings considered in [9] are used in the present algorithm to avoid equivalent patterns. As first-order non-guillotine cuts in which a rectangle is cut in exactly three or four subrectangles can be generated by two or three consecutive guillotine cuts (see [9] for details), only guillotine cuts (lines 18-31) and first-order non-guillotine cuts that generates exactly five subrectangles (lines 8-17) need to be considered in Algorithm 1.

In the Solve procedure, we note that if the depth limit $N$ is sufficiently large, the Five-block

\footnotetext{
${ }^{2}$ It is worth mentioning that there is a minor typographical error in line 13 of Algorithm 2 in [9] (which corresponds to Algorithm 1 here), although the computer implementation is correct - the correct constraint should be $y_{1}<y_{2}$ and $y_{2}<W$, instead of $y_{1}<y_{2}$ and $y_{1}+y_{2} \leq W$.
} 


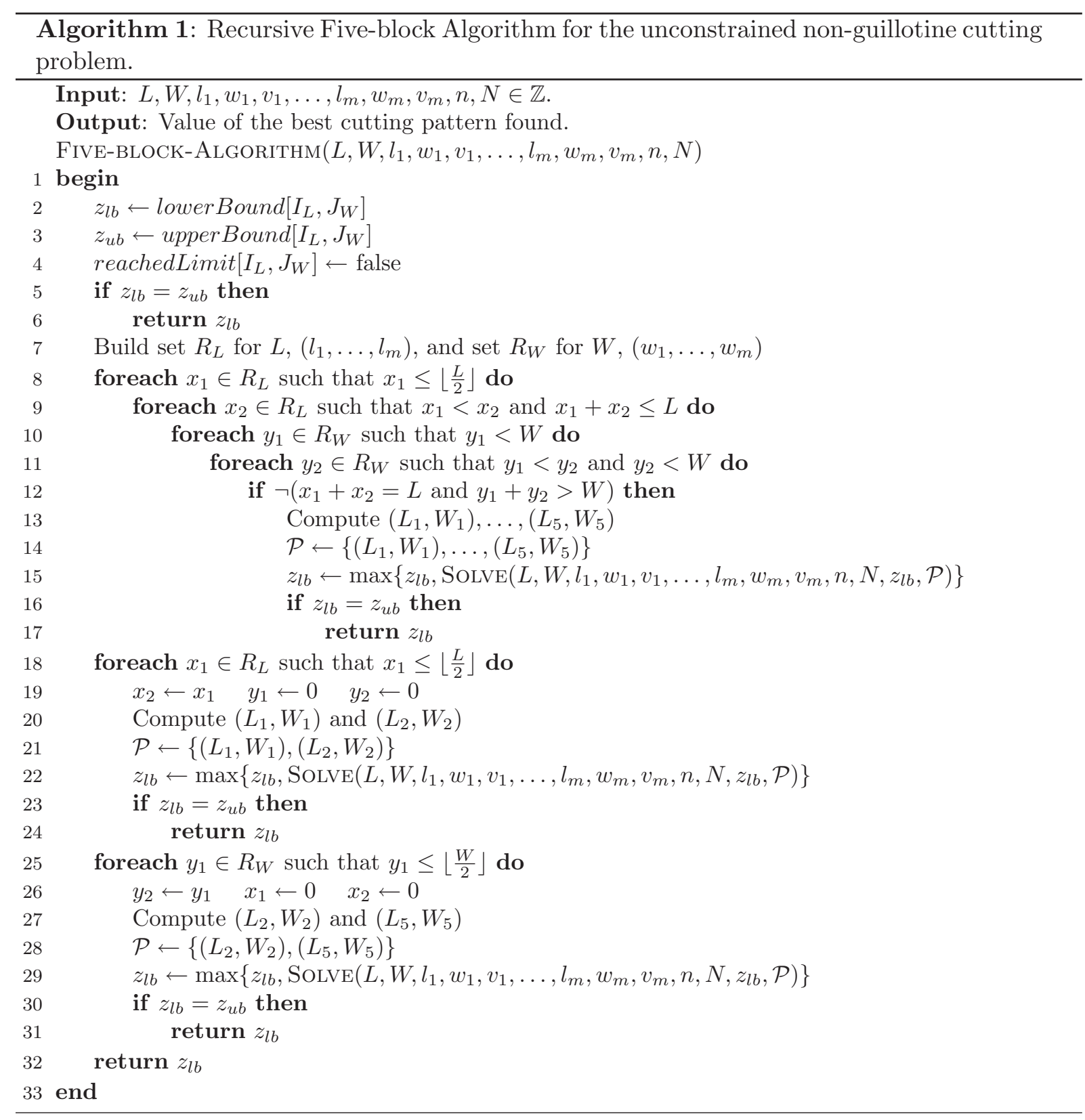

Algorithm is able to find the optimal first-order non-guillotine cutting pattern. Otherwise, by choosing small values of $N$, it is possible to control the tradeoff between the computer runtime and the quality of the solution found.

The same subproblem may appear in different levels of the tree search. In the case $N$ is not sufficiently large, a subproblem may be solved more than once (if its solution is not proven to be optimal) in order to try to improve the previous solution found for it. In this case, if the depth $n$ at which a subproblem is faced is greater than or equal to the depth of its stored solution, 


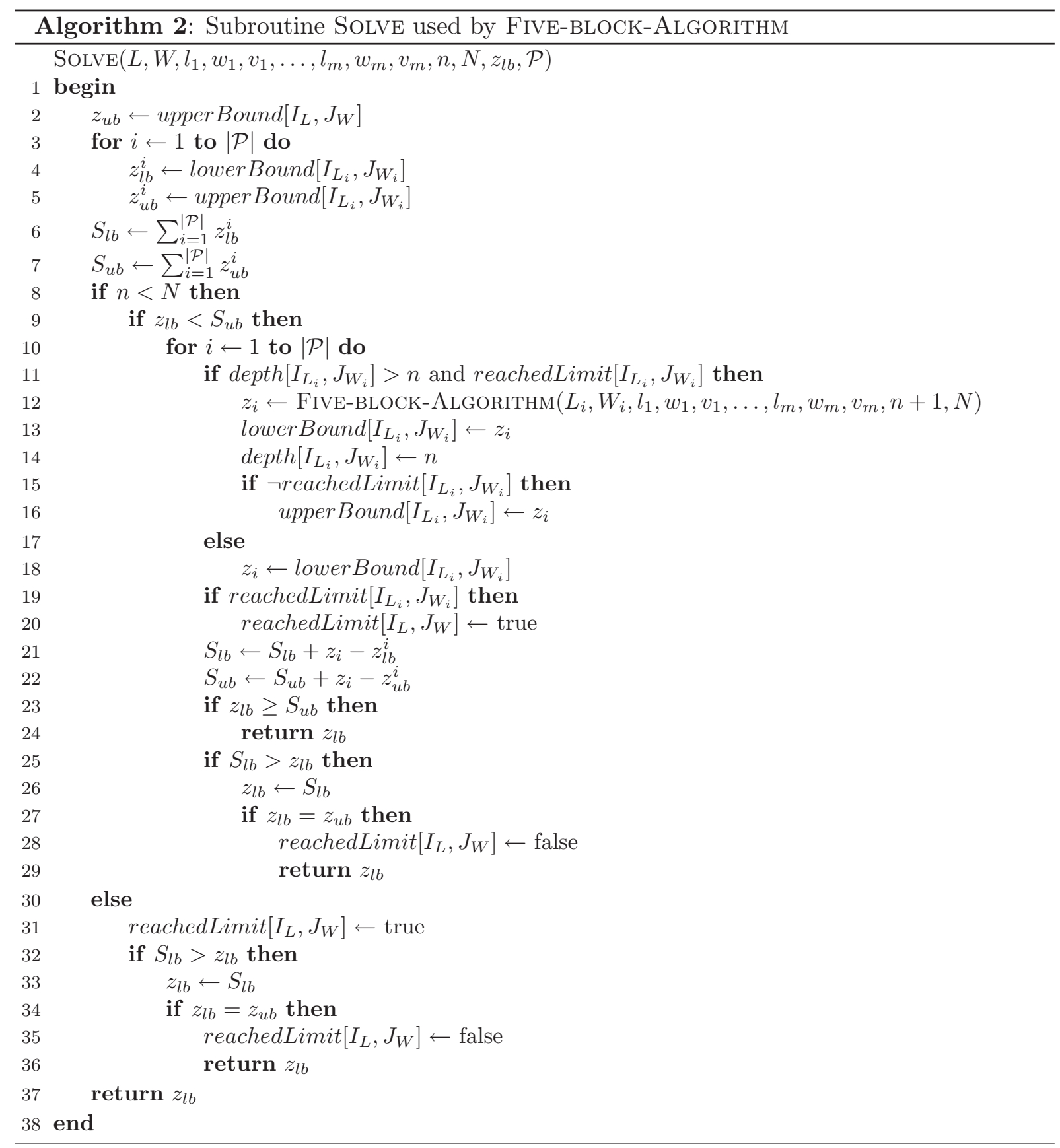

the subproblem is not solved again and the stored solution is used. Otherwise, if the current depth is smaller, there are two possibilities. If the depth limit $N$ was used to stop the recursion when obtaining the stored subproblem solution, then it is worth trying to solve it again. On the other hand, if the depth limit did not interfere in the subproblem resolution, the subproblem will not be solved again, as a better solution cannot be found. A key subject of the method 
being proposed is the storage of information of the subproblems previously considered by the algorithm during the execution. The considered data structure is dynamic and depends on the available memory, balancing space and time complexities. Raster points are associated with indices and a combination of arrays and balanced binary search trees is used to store a solution represented by a pair (five-block heuristic) or a quadruple ( $L$-approach) of raster points (see [9] (p. 313) for details). In Algorithm 1 (lines 2-4), $I_{X}$ represents the index associated with the raster point $X \in R_{L}$ and, analogously, $J_{Y}$ represents the index associated with the raster point $Y \in R_{W}$. More explicitly, if the sets of raster points $R_{L}$ and $R_{W}$ are considered ordered sets, the index of a raster point corresponds to its relative position within the set.

Using the same reasoning as in [9] for the manufacturer's pallet loading, it can be shown that the worst-case time complexity of the Five-block Heuristic for the unconstrained twodimensional non-guillotine cutting is $O\left(\left|R_{L}\right|\left|R_{W}\right|\left(\left|R_{L}\right|^{2}\left|R_{W}\right|^{2}+\left|S_{L}\right|+\left|S_{W}\right|\right)+L+W\right)$ and the memory complexity is $O\left(\left|R_{L}\right|\left|R_{W}\right|+L+W\right)$.

\subsection{Phase 2}

Phase 2 of the Recursive Partitioning Approach applies the $L$-approach $[37,12,9]$ which is based on the computation of a dynamic programming recursive formula [37]. This procedure divides a rectangle or an $L$-shaped piece into two $L$-shaped pieces. An $L$-shaped piece is determined by a quadruple $(X, Y, x, y)$, with $X \geq x$ and $Y \geq y$, and is defined as the topological closure of the rectangle whose diagonal goes from $(0,0)$ to $(X, Y)$ minus the rectangle whose diagonal goes from $(x, y)$ to $(X, Y)$. Figure 2 depicts the nine possible divisions [9] of a rectangle or an $L$-shaped piece into two $L$-shaped pieces.

A pseudo-code of the $L$-Algorithm for the unconstrained non-guillotine cutting problem is presented in Algorithms 3 and 4. In subroutine Solve-L (Algorithm 4), $\mathscr{L}_{i}\left(L, k, i_{k}\right)$ and $I_{k}(L)$, for $i=1,2$ and $k=1, \ldots, 9$, are defined as in [37,9]. Basically, each set $I_{k}(L)$, for $k=1, \ldots, 9$, contains all the pairs or triplets of raster points associated with the cut of type $k$ (the nine cut types are depicted in Figure 2) that divides a rectangle or an $L$-shaped piece into two $L$-shaped pieces, namely $\mathscr{L}_{1}\left(L, k, i_{k}\right)$ and $\mathscr{L}_{2}\left(L, k, i_{k}\right)$. More precisely, $\mathscr{L}_{1}\left(L, k, i_{k}\right)$ and $\mathscr{L}_{2}\left(L, k, i_{k}\right)$ represent the normalized $L$-shaped pieces obtained in the division process. Similarly to Algorithm 1, input parameters of $L$-Algorithm include the dimensions of the plate and the pieces, the current depth $n$ and the maximum depth limit $N$ of the search. As Algorithm 1, the $L$-Algorithm takes into account the storage of information of the subproblems previously solved during its execution. The same data structure used in [9] to store this information is used in the implementation of the present algorithm (a four dimensional array or a combination of an array and a balanced binary search tree). See [9] for details.

In the $L$-Algorithm, the lower bound for a rectangle $(X, Y)$ is given, initially, by the solution found in phase 1 for the subproblem $(X, Y)$. As the algorithm proceeds, the lower bound for the subproblem $(X, Y)$ may be improved and updated, so that the lower bound for $(X, Y)$ in future requests is no longer the solution found in the phase 1 , but the best solution found so far for the subproblem $(X, Y)$. A lower bound for a non-degenerate $L$-shaped piece can be computed by dividing it into two rectangles and summing up the lower bounds of these two rectangles. The two straightforward different ways of dividing an $L$-shaped piece into two rectangles are considered and the lower bound for the $L$-shaped piece is given by the best one out of these two 

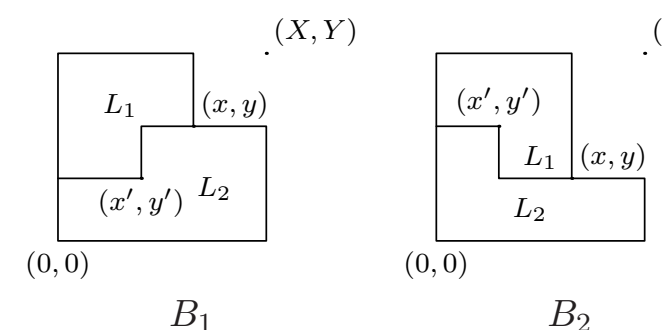

$(X, Y)$

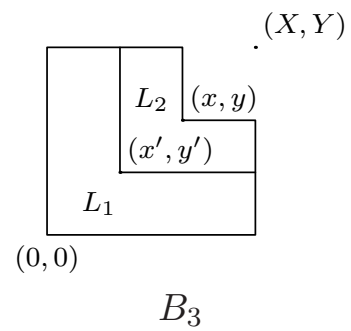

$(X, Y)$
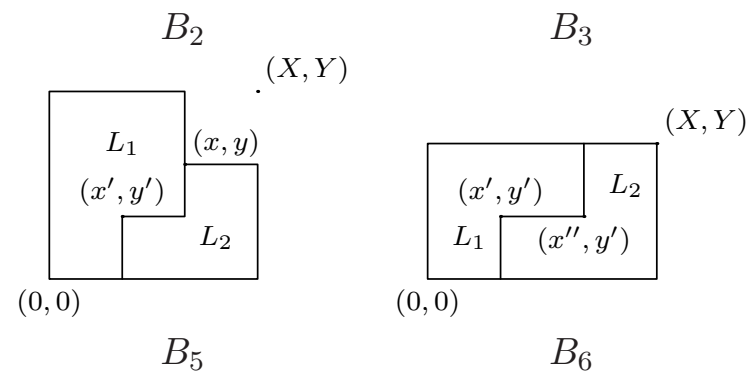

$(0,0)$

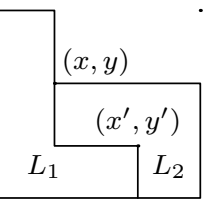

$B_{4}$

$(X, Y)$
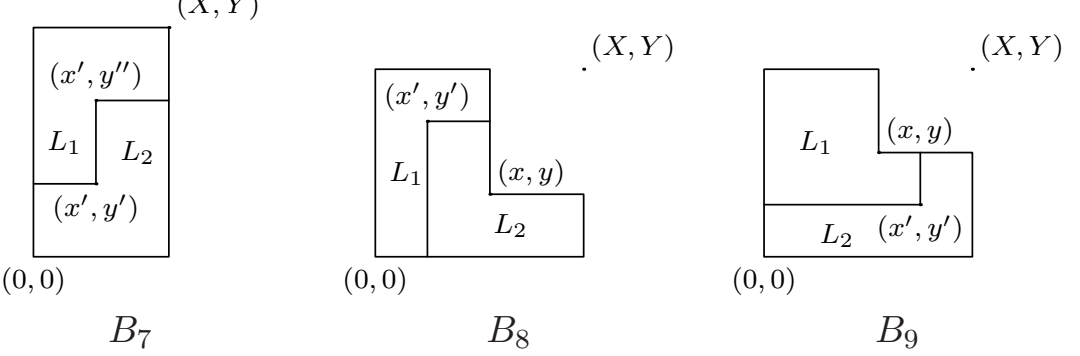

Figure 2: Subdivisions of an $L$-shaped piece into two $L$-shaped pieces.

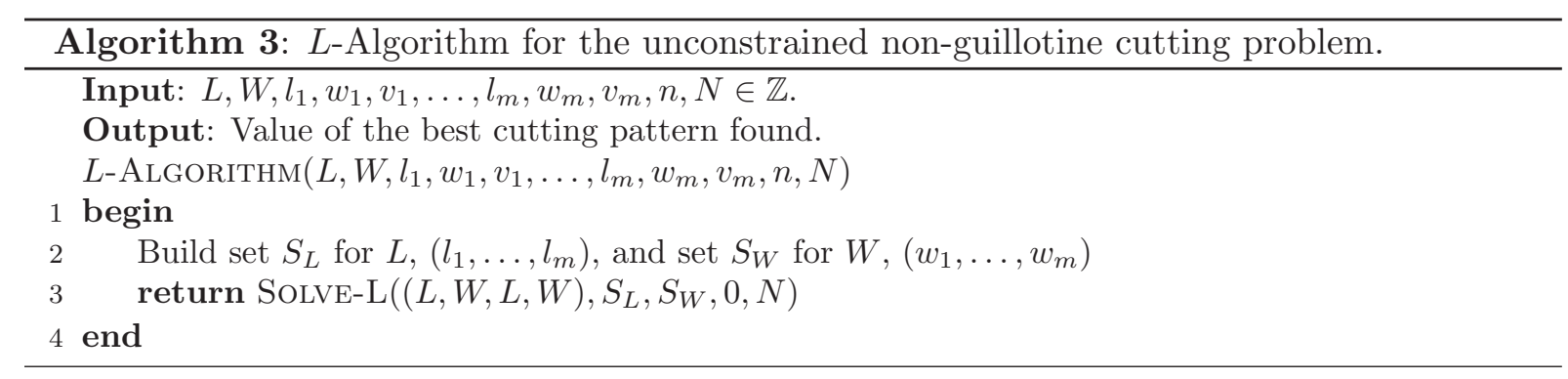

ones. As the algorithm proceeds, the lower bounds for $L$-shaped pieces are similarly updated as those for rectangles.

The upper bound for a rectangle $(X, Y)$ is the same one used in the previous phase, that is, it is computed by (6). The upper bound for a non-degenerate $L$-shaped piece is given by the area ratio. Among all pieces that fit into the $L$-shaped piece, the one with the greatest value by area ratio is considered. The upper bound for the $L$-shaped piece is then given by its area 


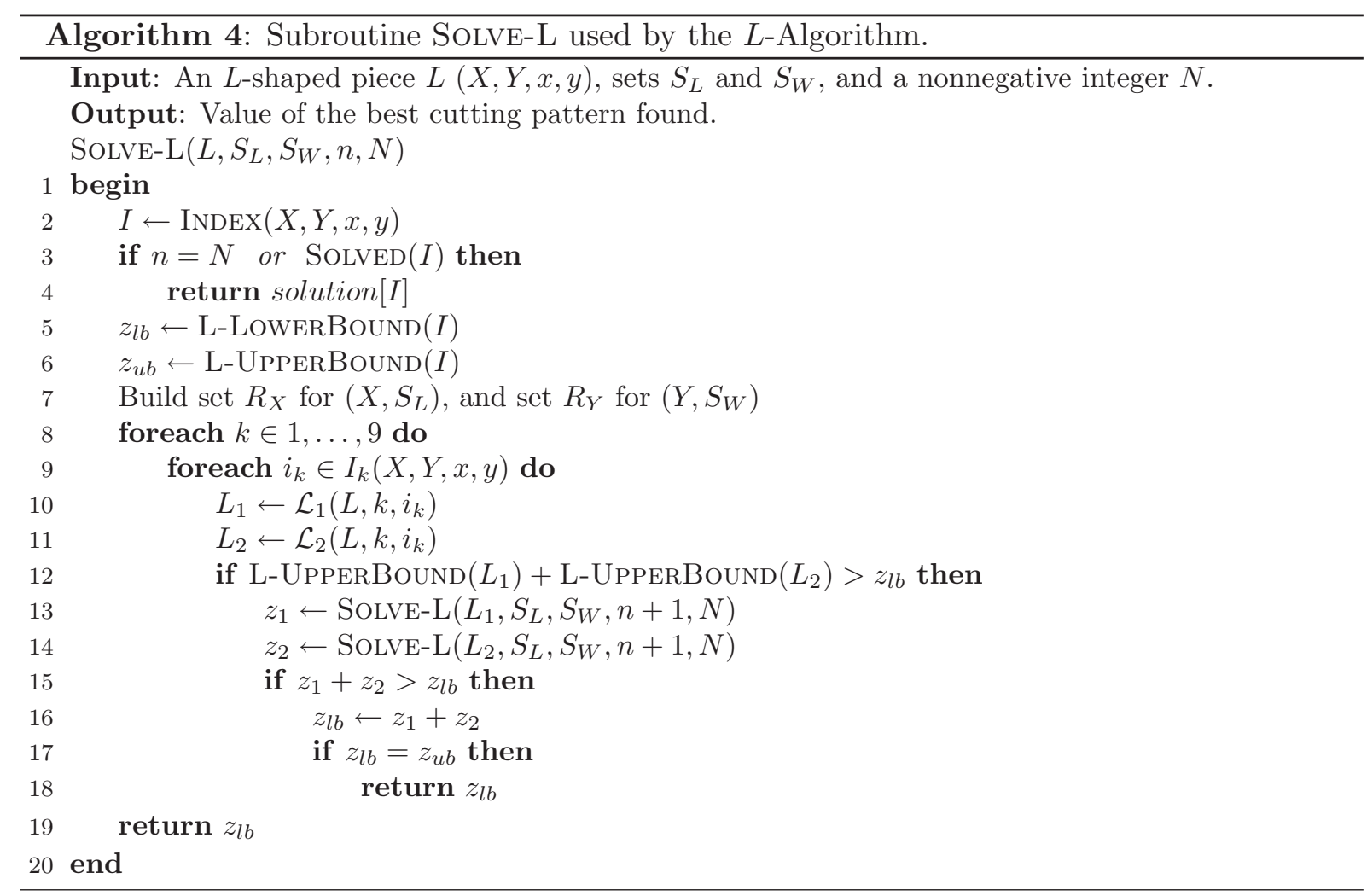

multiplied by the value per unit area of the most valuable piece, that is,

$$
\left\lfloor[X Y-(X-x)(Y-y)] \max _{i \in G(X, Y, x, y)}\left\{\frac{v_{i}}{l_{i} w_{i}}\right\}\right\rfloor,
$$

where $G(X, Y, x, y)=\{i \mid i \in F(X, y)$ or $i \in F(x, Y)\}$.

Following the same steps in [9] for the manufacturer's pallet loading, it can be shown that the worst-case time complexity of the $L$-Algorithm for the unconstrained two-dimensional nonguillotine cutting is

$$
O\left(\left|R_{L}\right|^{2}\left|R_{W}\right|^{2}\left(\left|R_{L}\right|\left|R_{W}\right|+\left|S_{L}\right|+\left|S_{W}\right|\right)+L+W\right)
$$

and the memory complexity is

$$
O\left(\left|R_{L}\right|^{2}\left|R_{W}\right|^{2}+L+W\right) .
$$

\subsection{Improving the lower bound and problem preprocessing}

In order to improve the computational performance of the Recursive Partitioning Approach, we start computing better lower bounds (feasible solutions) than the ones provided by the homogeneous solutions (5). The idea is to generate cutting patterns simpler than generic unconstrained non-guillotine cutting patterns, however, more elaborated than homogeneous patterns. 
In this way, before performing phase 1 of Section 3.1, the approach computes optimal unconstrained two-staged guillotine cutting patterns for the plate $(L, W)$ and for a number of subrectangles of $(L, W)$. This computation uses the exact procedure in [26], based on a solution of $O(m)$ one-dimensional knapsack problems. Then, the approach computes optimal non-staged guillotine cutting patterns for the plate $(L, W)$ and a number of subrectangles of $(L, W)$, using the value of the two-staged solutions as initial lower bounds. This computation employs the Recursive Five-block Algorithm specialized for the guillotine case (i.e., considering only vertical and horizontal guillotine cuts). These optimal guillotine solutions are used to reduce the tree search of the Recursive Five-block Heuristic in phase 1. Finally, the optimal first-order non-guillotine solutions found in phase 1 for the plate $(L, W)$ and a number of subrectangles of $(L, W)$ are used to reduce the dynamic programming recursions of the $L$-approach in phase 2 (Section 3.2). We note that each phase of the approach is executed only if the solution given by the previous phase cannot be proven to be optimal. Solutions found for the subproblems in a given phase are used as lower bounds for the same subproblems in the next phase. In this way, solutions found in higher phases are at least as good as the ones found in earlier phases.

Using the same reasoning as in [9] for the manufacturer's pallet loading, it can be shown that the worst-case time complexity of computing optimal unconstrained two-staged guillotine cutting patterns is $O\left(m^{2}\left(\left|S_{L}\right|+\left|S_{W}\right|\right)+L+W\right)$ and the memory complexity is $O\left(m\left(\left|S_{L}\right|+\left|S_{W}\right|\right)+L+\right.$ $W)$. The worst-case time and memory complexities of computing optimal non-staged guillotine cutting patterns are $O\left(\left|R_{L}\right|\left|R_{W}\right|\left(\left|S_{L}\right|+\left|S_{W}\right|\right)+L+W\right)$ and $O\left(\left|R_{L}\right|\left|R_{W}\right|+L+W\right)$, respectively. Summing up the worst-case time and space complexities of phases 1 and 2 and the ones of improving the lower bounds, we have that the worst-case time and memory complexities of the Recursive Partitioning Approach are given by $O\left(\left|R_{L}\right|^{3}\left|R_{W}\right|^{3}+\left(\left|R_{L}\right|^{2}\left|R_{W}\right|^{2}+m^{2}\right)\left(\left|S_{L}\right|+\left|S_{W}\right|\right)+\right.$ $L+W)$ and $O\left(\left|R_{L}\right|^{2}\left|R_{W}\right|^{2}+m\left(\left|S_{L}\right|+\left|S_{W}\right|\right)+L+W\right)$, respectively.

A final remark in this section is that as a preprocessing of the input of the Recursive Partitioning Algorithm, our implementation of the algorithm may discard pieces that are not required to be in an optimal solution. Note that any piece type $i$ that satisfies $l_{i} \geq l_{j}, w_{i} \geq w_{j}$, and $v_{i} \leq v_{j}$, for some piece type $j \neq i$, does not need to be considered, since $j$ is at least as valuable as $i$ and it is not larger than $i$. If this is the case, we say that piece type $j$ dominates piece type $i$. It is worth mentioning that this procedure may be useful for weighted instances only. This process could be generalized to the case where not only one, but a combination of pieces dominates another piece. That is, when there is a subset of pieces $P$ and a piece $i \notin P$, such that we can pack all pieces that belong to $P$ (maybe using more than one of the same type) in rectangle $i$, and the packing has value greater than or equal to $v_{i}$. For unweighted instances, if a piece $i$ is dominated by pieces in set $P$, then there is a packing of the pieces in $P$ in the rectangle $i$ so that rectangle $i$ is completely covered (i.e, a zero waste packing). Hence, all integer conic combinations produced by piece $i$ are also produced by the ones in the set $P$ and, therefore, the exclusion of piece $i$ does not contribute to a reduction on the number of patterns that the algorithm must inspect. As the generalized process described above is a computational expensive task, in the present work we implemented a preprocessing procedure that considers the elimination of a piece dominated by another piece, whose worst-case time complexity is $O\left(\mathrm{~m}^{2}\right)$. 


\subsection{Heuristics for large problems}

The generation of all patterns by the Recursive Partitioning Approach may be prohibitive for large instances. Moreover, the amount of memory required by these algorithms may not be available. For this reason, we propose heuristics that reduce both the time and memory requirements of the algorithms. These procedures, however, may lead to a loss of quality of the solution found. Since the time and memory complexities of generating all possible cuttings highly depends on the sizes of the integer conic combinations and raster points sets (as pointed out in (7) and (8), respectively), we can significantly reduce time and memory requirements in two ways: (i) by limiting the search depth of the recursions (i.e., setting parameter $N \neq \infty$ in Algorithms 1-4); and (ii) by replacing the integer conic combinations and raster points sets by smaller sets.

In order to reduce the sets of conic combinations $S_{L}$ and $S_{W}$, it was suggested in [6] a simple iterative procedure that starts eliminating the piece with the smallest length (width) until the desired sizes of the sets are achieved. In this work we decided by an alternative procedure that replaces the sets $S_{L}$ and $S_{W}$ by the shrunk sets $\tilde{S}_{L}$ and $\tilde{S}_{W}$, so that the product $\left|\tilde{S}_{L}\right|\left|\tilde{S}_{W}\right|$ is limited by a given threshold parameter $M$. Moreover, to substitute the raster points sets, the shrunk sets $\tilde{R}_{L}$ and $\tilde{R}_{W}$ are obtained by using definition (4) with $\tilde{S}_{L}$ and $\tilde{S}_{W}$ instead of $S_{L}$ and $S_{W}$.

Algorithms 5-7 describe the implemented reduction strategy. Basically, the strategy consists in selecting equally distributed elements within ordered versions of sets $S_{L}$ and $S_{W}$. Algorithm 7 describes the main routine named REDUCESETs that uses Algorithms 5 and 6 as subroutines. Specifically, the heuristic version of the Recursive Partitioning Algorithm makes use of routine REDUCESETS in line 7 of Algorithm 1 to compute $\tilde{R}_{L}$ and $\tilde{R}_{W}$ based on $\tilde{S}_{L}$ and $\tilde{S}_{W}$. Similarly, Algorithm 3 uses routine REDUCESETS in line 2 to build sets $\tilde{S}_{L}$ and $\tilde{S}_{W}$ and call subroutine Solve-L (Algorithm 4) in line 3 with them, instead of $S_{L}$ and $S_{W}$.

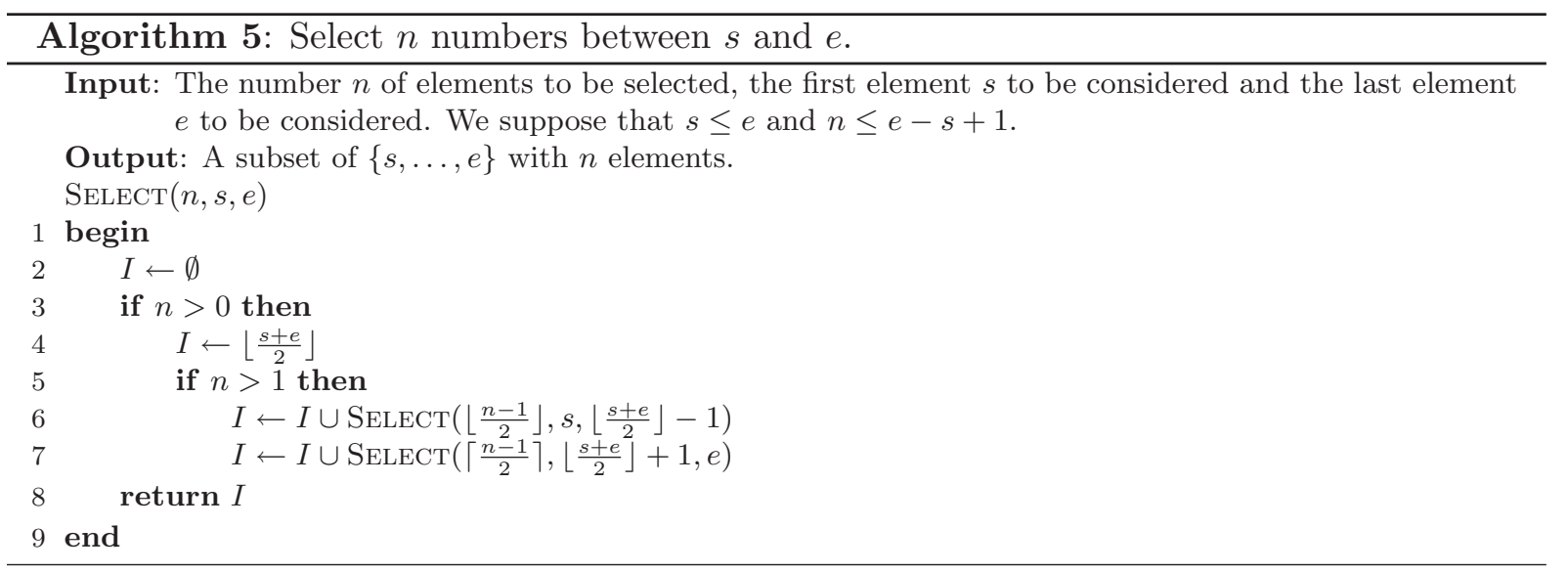



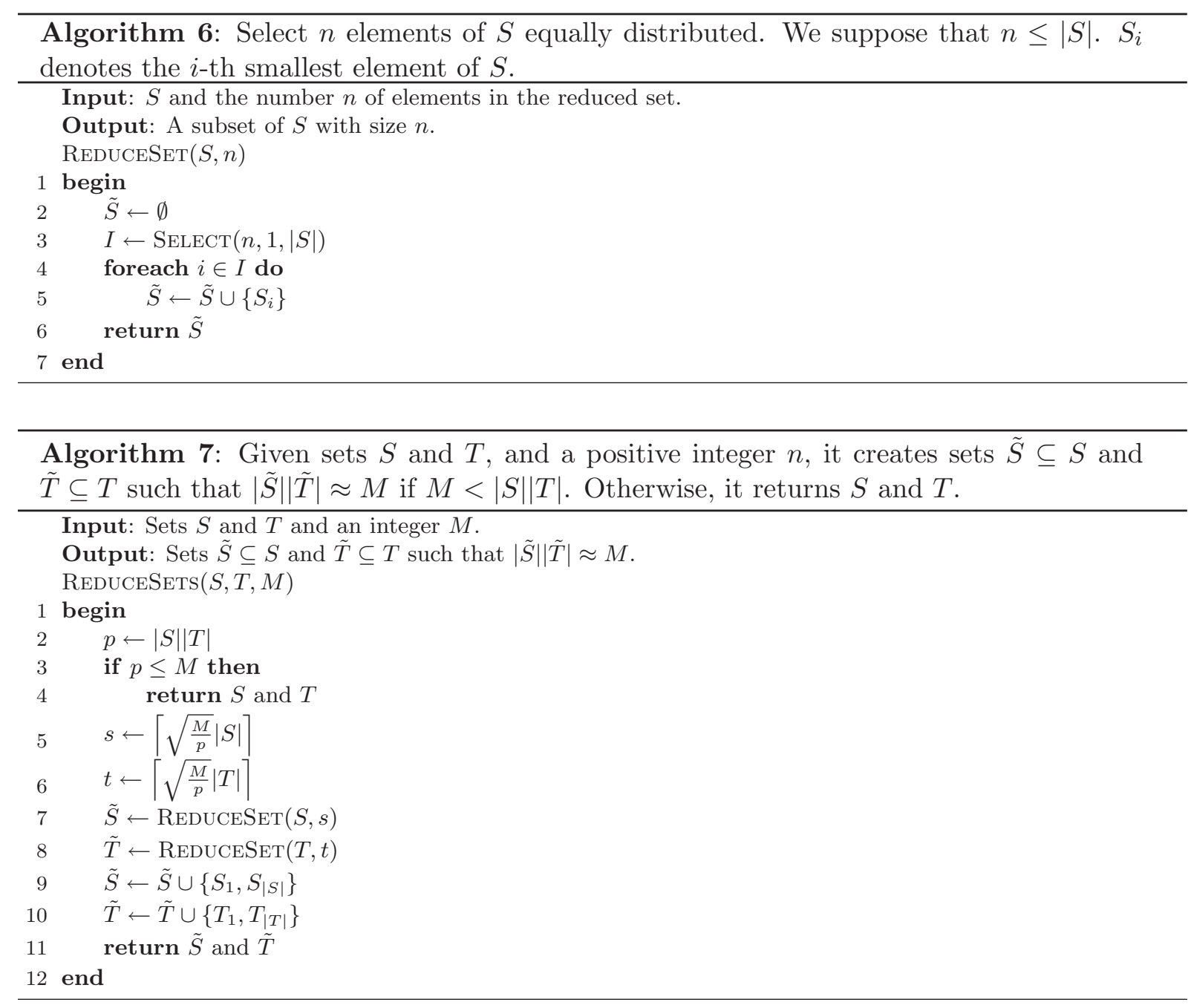

\section{Numerical experiments}

We implemented the Recursive Partitioning Approach and its heuristic counterpart for the unconstrained two-dimensional non-guillotine cutting problem as described in Algorithms 1-7. The algorithms were coded in $\mathrm{C} / \mathrm{C}++$ language. The experiments were performed in a $2.4 \mathrm{GHz}$ Intel Core 2 Quad Q6600 with 8.0GB of RAM memory and Linux Operating System. Compiler option -O3 has been adopted. The computer implementation of the algorithms as well as the data sets used in our experiments and the solutions found are publicly available for benchmarking purposes at [49].

In the numerical experiments, we considered 95 problem instances found in the literature: $\mathrm{H}$, HZ1 and HZ2 from [29], [32] and [33], respectively; GCUT1-GCUT13 and GCUT14-GCUT17 from [5] and [18], respectively; M1-M5 and MW1-MW4 from [39] and [31], respectively; U1-U3 and W1-W3 from [30]; U4, W4, LU1-LU4, LW1-LW4 from [31]; UU1-UU11 and UW1-UW11 from [23]; APT10-APT29 from [1]; and B1-B7 from [19]. Table 1 presents some characteristics 
of these instances. In the table, $m$ is the original number of piece types while $m_{\mathrm{r}}$ is the reduced number of piece types obtained by applying the preprocessing procedure described in Subsection 3.3, $L$ and $W$ are the length and width of the stock plate, and $\left|S_{L}\right|,\left|S_{W}\right|,\left|R_{L}\right|$ and $\left|R_{W}\right|$ are the number of elements of the sets of conic combinations and raster points, respectively, after applying the preprocessing procedure for the elimination of dominated pieces described in Subsection 3.3. It should be noted in Table 1 that the number of piece types of the instances varies from 5 to 200 and the number of conic combinations (or raster points) from a few tens to thousands. Therefore, the number of variables and constraints of model (1-3) for these instances varies from a few hundreds to billions.

We arbitrarily divided the experiments with these instances into two parts: the first consists of experiments with instances of moderate size, defined as $\max \left\{\left|S_{L}\right|,\left|S_{W}\right|\right\}<1,000$, and the second part contains experiments with the remaining large-sized instances.

\begin{tabular}{|c|c|c|c|c|c|c|c|c|c|}
\hline Instance & $m_{\mathrm{r}}$ & $m$ & $L$ & $W$ & $\left|S_{L}\right|$ & $\left|S_{W}\right|$ & $\left|R_{L}\right|$ & $\left|R_{W}\right|$ & $\left|S_{L}\right|\left|S_{W}\right|$ \\
\hline $\mathrm{H}$ & 5 & 5 & 127 & 98 & 35 & 61 & 25 & 30 & 2135 \\
\hline HZ1 & 6 & 6 & 78 & 67 & 70 & 44 & 61 & 23 & 3080 \\
\hline $\mathrm{HZ} 2$ & 5 & 5 & 99 & 80 & 25 & 56 & 17 & 31 & 1400 \\
\hline GCUT1 & 10 & 10 & 250 & 250 & 68 & 20 & 13 & 5 & 1360 \\
\hline GCUT2 & 20 & 20 & 250 & 250 & 95 & 112 & 17 & 24 & 10640 \\
\hline GCUT3 & 30 & 30 & 250 & 250 & 143 & 107 & 44 & 26 & 15301 \\
\hline GCUT4 & 50 & 50 & 250 & 250 & 146 & 146 & 45 & 50 & 21316 \\
\hline GCUT5 & 10 & 10 & 500 & 500 & 40 & 76 & 10 & 13 & 3040 \\
\hline GCUT6 & 20 & 20 & 500 & 500 & 96 & 120 & 12 & 18 & 11520 \\
\hline GCUT7 & 30 & 30 & 500 & 500 & 179 & 126 & 23 & 19 & 22554 \\
\hline GCUT8 & 50 & 50 & 500 & 500 & 225 & 262 & 44 & 59 & 58950 \\
\hline GCUT9 & 10 & 10 & 1000 & 1000 & 92 & 42 & 15 & 7 & 3864 \\
\hline GCUT10 & 20 & 20 & 1000 & 1000 & 89 & 155 & 14 & 20 & 13795 \\
\hline GCUT11 & 30 & 30 & 1000 & 1000 & 238 & 326 & 20 & 38 & 77588 \\
\hline GCUT12 & 50 & 50 & 1000 & 1000 & 398 & 363 & 49 & 42 & 144474 \\
\hline GCUT13 & 32 & 32 & 3000 & 3000 & 1821 & 2425 & 647 & 1849 & 4415925 \\
\hline GCUT14 & 42 & 42 & 3500 & 3500 & 2681 & 2976 & 1861 & 2451 & 7978656 \\
\hline GCUT15 & 52 & 52 & 3500 & 3500 & 2690 & 3048 & 1879 & 2595 & 8199120 \\
\hline GCUT16 & 62 & 62 & 3500 & 3500 & 2824 & 3058 & 2147 & 2615 & 8635792 \\
\hline GCUT17 & 82 & 82 & 3500 & 3500 & 2929 & 3068 & 2357 & 2635 & 8986172 \\
\hline M1 & 10 & 10 & 100 & 156 & 48 & 74 & 23 & 17 & 3552 \\
\hline M2 & 10 & 10 & 253 & 294 & 154 & 87 & 63 & 17 & 13398 \\
\hline M3 & 10 & 10 & 318 & 473 & 72 & 156 & 13 & 32 & 11232 \\
\hline M4 & 10 & 10 & 501 & 556 & 78 & 116 & 15 & 15 & 9048 \\
\hline M5 & 10 & 10 & 750 & 806 & 124 & 147 & 23 & 16 & 18228 \\
\hline MW1 & 10 & 10 & 100 & 156 & 48 & 74 & 23 & 17 & 3552 \\
\hline MW2 & 9 & 10 & 253 & 294 & 145 & 87 & 53 & 17 & 12615 \\
\hline MW3 & 8 & 10 & 318 & 473 & 60 & 130 & 13 & 28 & 7800 \\
\hline MW4 & 9 & 10 & 501 & 556 & 106 & 112 & 22 & 15 & 11872 \\
\hline MW5 & 8 & 10 & 750 & 806 & 99 & 100 & 22 & 12 & 9900 \\
\hline U1 & 10 & 10 & 4500 & 5000 & 2910 & 1984 & 1327 & 253 & 5773440 \\
\hline U2 & 10 & 10 & 5050 & 4070 & 2225 & 373 & 351 & 38 & 829925 \\
\hline U3 & 20 & 20 & 7350 & 6579 & 3948 & 3711 & 832 & 979 & 14651028 \\
\hline U4 & 40 & 40 & 7350 & 6579 & 6151 & 4898 & 4951 & 3216 & 30127598 \\
\hline W1 & 6 & 20 & 5000 & 5000 & 1288 & 1228 & 190 & 179 & 1581664 \\
\hline W2 & 9 & 20 & 3427 & 2769 & 407 & 382 & 60 & 58 & 155474 \\
\hline W3 & 7 & 40 & 7500 & 7381 & 1029 & 1477 & 129 & 180 & 1519833 \\
\hline W4 & 12 & 80 & 7500 & 7381 & 5412 & 5494 & 3323 & 3606 & 29733528 \\
\hline UU1 & 25 & 25 & 500 & 500 & 171 & 205 & 29 & 39 & 35055 \\
\hline UU2 & 30 & 30 & 750 & 800 & 334 & 322 & 61 & 38 & 107548 \\
\hline UU3 & 25 & 25 & 1100 & 1000 & 389 & 317 & 44 & 37 & 123313 \\
\hline UU4 & 38 & 38 & 1000 & 1200 & 444 & 570 & 65 & 83 & 253080 \\
\hline UU5 & 50 & 50 & 1450 & 1300 & 694 & 643 & 111 & 116 & 446242 \\
\hline UU6 & 38 & 38 & 2050 & 1457 & 649 & 495 & 59 & 50 & 321255 \\
\hline UU7 & 50 & 50 & 1465 & 2024 & 743 & 944 & 144 & 139 & 701392 \\
\hline UU8 & 55 & 55 & 2000 & 2000 & 914 & 830 & 114 & 95 & 758620 \\
\hline UU9 & 60 & 60 & 2500 & 2460 & 1047 & 1044 & 120 & 127 & 1093068 \\
\hline UU10 & 55 & 55 & 3500 & 3450 & 1304 & 1542 & 110 & 177 & 2010768 \\
\hline UU11 & 25 & 25 & 3500 & 3765 & 3014 & 2461 & 2527 & 1156 & 7417454 \\
\hline
\end{tabular}

Table 1: Characteristics of the instances. 


\begin{tabular}{|c|c|c|c|c|c|c|c|c|c|}
\hline Instance & $m_{\mathrm{r}}$ & $m$ & $L$ & $W$ & $\left|S_{L}\right|$ & $\left|S_{W}\right|$ & $\left|R_{L}\right|$ & $\left|R_{W}\right|$ & $\left|S_{L}\right|\left|S_{W}\right|$ \\
\hline UW1 & 7 & 25 & 500 & 500 & 72 & 70 & 10 & 18 & 5040 \\
\hline UW2 & 8 & 35 & 560 & 750 & 172 & 80 & 31 & 16 & 13760 \\
\hline UW3 & 9 & 35 & 700 & 650 & 96 & 98 & 18 & 21 & 9408 \\
\hline UW4 & 10 & 45 & 1245 & 1015 & 193 & 251 & 23 & 33 & 48443 \\
\hline UW5 & 6 & 35 & 1100 & 1450 & 76 & 68 & 19 & 9 & 5168 \\
\hline UW6 & 15 & 47 & 1750 & 1542 & 310 & 379 & 37 & 38 & 117490 \\
\hline UW7 & 11 & 50 & 2250 & 1875 & 185 & 354 & 27 & 33 & 65490 \\
\hline UW8 & 13 & 55 & 2645 & 2763 & 450 & 540 & 49 & 39 & 243000 \\
\hline UW9 & 14 & 45 & 3000 & 3250 & 357 & 384 & 38 & 39 & 137088 \\
\hline UW10 & 17 & 60 & 3500 & 3650 & 690 & 797 & 61 & 53 & 549930 \\
\hline UW11 & 8 & 25 & 555 & 632 & 259 & 327 & 64 & 104 & 84693 \\
\hline LU1 & 97 & 100 & 20789 & 23681 & 10075 & 22602 & 9753 & 21522 & 227715150 \\
\hline LU2 & 98 & 100 & 25587 & 34563 & 24478 & 33421 & 23368 & 32278 & 818079238 \\
\hline LU3 & 149 & 150 & 37587 & 27563 & 37022 & 13684 & 36456 & 13584 & 506609048 \\
\hline LU4 & 200 & 200 & 45237 & 35983 & 22546 & 17894 & 22471 & 17794 & 403438124 \\
\hline LW1 & 56 & 100 & 20789 & 23681 & 10057 & 11516 & 9717 & 11189 & 115816412 \\
\hline LW2 & 48 & 100 & 25587 & 34563 & 12447 & 33403 & 12098 & 32242 & 415767141 \\
\hline LW3 & 83 & 150 & 37587 & 27563 & 18627 & 13677 & 18458 & 13570 & 254761479 \\
\hline LW4 & 112 & 200 & 45237 & 35983 & 22546 & 17883 & 22471 & 17772 & 403190118 \\
\hline APT10 & 51 & 51 & 2097 & 1713 & 1760 & 1478 & 1422 & 1242 & 2601280 \\
\hline APT11 & 58 & 58 & 2600 & 1612 & 2156 & 1435 & 1711 & 1257 & 3093860 \\
\hline APT12 & 35 & 35 & 2662 & 1941 & 1966 & 1420 & 1269 & 898 & 2791720 \\
\hline APT13 & 54 & 54 & 1674 & 2090 & 1422 & 1802 & 1169 & 1513 & 2562444 \\
\hline APT14 & 42 & 42 & 2090 & 2138 & 1739 & 1681 & 1387 & 1223 & 2923259 \\
\hline APT15 & 49 & 49 & 2222 & 2726 & 1741 & 2264 & 1259 & 1801 & 3941624 \\
\hline APT16 & 53 & 53 & 2899 & 2614 & 2292 & 2104 & 1684 & 1593 & 4822368 \\
\hline APT17 & 59 & 59 & 2313 & 1962 & 1980 & 1643 & 1646 & 1323 & 3253140 \\
\hline APT18 & 31 & 31 & 2775 & 2105 & 2163 & 1524 & 1550 & 942 & 3296412 \\
\hline APT19 & 35 & 35 & 2284 & 2994 & 1729 & 2325 & 1173 & 1655 & 4019925 \\
\hline АРТ 20 & 34 & 45 & 2840 & 2858 & 2284 & 2260 & 1727 & 1661 & 5161840 \\
\hline APT 21 & 34 & 48 & 2866 & 1784 & 2066 & 1417 & 1265 & 1049 & 2927522 \\
\hline APT 22 & 37 & 52 & 2711 & 2110 & 2168 & 1708 & 1624 & 1305 & 3702944 \\
\hline АРТ 23 & 35 & 53 & 1856 & 2636 & 1401 & 2048 & 945 & 1459 & 2869248 \\
\hline APT 24 & 33 & 44 & 2070 & 2729 & 1632 & 2308 & 1193 & 1886 & 3766656 \\
\hline APT 25 & 24 & 36 & 2885 & 1715 & 2246 & 1307 & 1606 & 898 & 2935522 \\
\hline APT 26 & 34 & 48 & 2359 & 1656 & 1842 & 1340 & 1324 & 1023 & 2468280 \\
\hline АРТ 27 & 33 & 48 & 1793 & 1875 & 1429 & 1397 & 1064 & 918 & 1996313 \\
\hline APT28 & 36 & 54 & 2020 & 2796 & 1604 & 2383 & 1187 & 1969 & 3822332 \\
\hline АРT 29 & 41 & 59 & 1839 & 2829 & 1505 & 2261 & 1170 & 1692 & 3402805 \\
\hline B1 & 30 & 30 & 4000 & 2000 & 3452 & 1343 & 2903 & 685 & 4636036 \\
\hline B2 & 30 & 30 & 4000 & 2000 & 3325 & 1429 & 2649 & 857 & 4751425 \\
\hline B3 & 30 & 30 & 4000 & 2000 & 3370 & 1411 & 2739 & 821 & 4755070 \\
\hline B4 & 30 & 30 & 4000 & 2000 & 3388 & 1426 & 2775 & 851 & 4831288 \\
\hline B5 & 30 & 30 & 4000 & 2000 & 3324 & 1393 & 2647 & 785 & 4630332 \\
\hline B6 & 30 & 30 & 4000 & 2000 & 3299 & 1441 & 2597 & 881 & 4753859 \\
\hline B7 & 180 & 180 & 4000 & 2000 & 3654 & 1664 & 3307 & 1327 & 6080256 \\
\hline
\end{tabular}

Table 1: Characteristics of the instances (cont.).

\subsection{Instances of moderate size}

In this set of experiments, we imposed no limit to the search depth, i.e., we set $N=\infty$. Table 2 presents the values of the unconstrained two-stage, guillotine, first-order non-guillotine (Five-block Algorithm) and (superior-order) non-guillotine ( $L$-algorithm) cutting patterns obtained by the Recursive Partitioning Approach for the instances of moderate size. The twostage and guillotine solutions are computed before phase 1 to obtain non-trivial lower bounds as described in Section 3.3. The Five-block Algorithm solution and the L-Algorithm solution correspond to phases 1 and 2 of the Recursive Partitioning Approach, respectively. The symbol "-" in the table means that an optimality certificate was found by closing the gap between the upper and the lower bound of the problem and, in consequence, the execution of the method was terminated. Columns with times represent the CPU time of each phase of the method. The time in the last column is the sum of the times of each phase plus the time used to read and preprocess the input data, compute lower and upper bounds for each possible subrectangle, draw and save the solution.

In the table, the numbers in bold indicate that the corresponding phase of the method 


\begin{tabular}{|c|c|c|c|c|c|c|c|c|c|}
\hline \multirow{2}{*}{ Instance } & \multicolumn{2}{|c|}{ Two-stage cuts } & \multicolumn{2}{|c|}{ Guillotine cuts } & \multicolumn{2}{|c|}{ Five-block Algorithm } & \multicolumn{2}{|c|}{$L$-Algorithm } & \multirow{2}{*}{$\begin{array}{l}\text { Total } \\
\text { time }\end{array}$} \\
\hline & Solution & Time $(\mathrm{sec})$ & Solution & Time $(\mathrm{sec})$ & Solution & Time $(\mathrm{sec})$ & Solution & Time (sec) & \\
\hline $\mathrm{H}$ & 12132 & 0.62 & 12348 & 0.00 & - & - & - & - & 1.67 \\
\hline HZ1 & 5226 & 0.67 & - & - & - & - & - & - & 1.30 \\
\hline $\mathrm{HZ} 2$ & 8046 & 0.57 & 8226 & 0.00 & 8443 & 0.01 & 8443 & 2.18 & 4.23 \\
\hline GCUT1 & 56460 & 0.57 & 56460 & 0.00 & 58480 & 0.00 & 58480 & 0.72 & 2.84 \\
\hline GCUT2 & 60076 & 0.69 & 60536 & 0.00 & 61146 & 0.00 & 61146 & 0.96 & 3.03 \\
\hline GCUT3 & 60133 & 0.58 & 61036 & 0.00 & 61275 & 0.02 & 61275 & 4.21 & 6.41 \\
\hline GCUT4 & 61698 & 0.70 & 61698 & 0.00 & 61918 & 0.06 & 61918 & 20.22 & 22.35 \\
\hline GCUT5 & 246000 & 0.54 & 246000 & 0.00 & 246000 & 0.00 & 246000 & 0.67 & 2.74 \\
\hline GCUT6 & 238998 & 0.71 & 238998 & 0.00 & 243598 & 0.00 & 243598 & 0.74 & 2.82 \\
\hline GCUT7 & 242567 & 0.56 & 242567 & 0.00 & 244306 & 0.00 & 244306 & 0.92 & 2.98 \\
\hline GCUT8 & 245758 & 0.73 & 246633 & 0.00 & 247815 & 0.10 & 247815 & 32.57 & 34.82 \\
\hline GCUT9 & 971100 & 0.54 & 971100 & 0.00 & 971100 & 0.00 & 971100 & 0.68 & 2.79 \\
\hline GCUT10 & 982025 & 0.71 & 982025 & 0.00 & 982025 & 0.00 & 982025 & 0.76 & 2.83 \\
\hline GCUT11 & 980096 & 0.56 & 980096 & 0.16 & 980096 & 0.00 & 980096 & 1.92 & 4.05 \\
\hline GCUT12 & 978776 & 0.53 & 979986 & 0.16 & 979986 & 0.06 & 979986 & 16.95 & 19.06 \\
\hline M1 & 15024 & 0.55 & 15024 & 0.00 & 15054 & 0.00 & 15073 & 1.13 & 3.20 \\
\hline M2 & 72172 & 0.66 & 73176 & 0.00 & 73255 & 0.04 & 73255 & 7.90 & 10.02 \\
\hline M3 & 141810 & 0.57 & 142817 & 0.00 & 147386 & 0.00 & 147386 & 1.10 & 3.16 \\
\hline M4 & 265768 & 0.47 & 265768 & 0.00 & 266233 & 0.00 & 266233 & 0.76 & 2.80 \\
\hline M5 & 577882 & 0.71 & 577882 & 0.00 & 579883 & 0.00 & 579883 & 1.08 & 3.14 \\
\hline MW1 & 3882 & 0.50 & 3882 & 0.00 & 3882 & 0.00 & 3882 & 1.22 & 3.23 \\
\hline MW2 & 24950 & 0.67 & 24950 & 0.00 & 24950 & 0.01 & 24950 & 6.17 & 8.24 \\
\hline MW3 & 37068 & 0.49 & 37068 & 0.00 & 37068 & 0.00 & 37068 & 1.01 & 3.04 \\
\hline MW4 & 59364 & 0.50 & 59576 & 0.00 & 59576 & 0.00 & 59576 & 1.07 & 3.05 \\
\hline MW5 & 189924 & 0.54 & 189924 & 0.00 & 189924 & 0.00 & 189924 & 0.94 & 2.98 \\
\hline W2 & 34520 & 0.70 & 35159 & 0.00 & 35822 & 0.96 & 35822 & 229.76 & 232.81 \\
\hline UU1 & 240346 & 0.58 & 242919 & 0.00 & 245205 & 0.02 & 245205 & 5.48 & 7.62 \\
\hline UU2 & 595288 & 0.70 & 595288 & 0.00 & 595288 & 0.08 & 595288 & 34.24 & 36.39 \\
\hline UU3 & 1065051 & 0.50 & 1072764 & 0.16 & 1088154 & 0.04 & 1088154 & 16.51 & 18.61 \\
\hline UU4 & 1177371 & 0.52 & 1179050 & 0.17 & 1191071 & 0.82 & 1191071 & 330.16 & 333.02 \\
\hline UU5 & 1868985 & 0.49 & 1868999 & 0.18 & 1870038 & 4.52 & 1870038 & 3057.23 & 3063.82 \\
\hline UU6 & 2950760 & 0.52 & 2950760 & 0.16 & 2950760 & 0.16 & 2950760 & 61.50 & 63.80 \\
\hline UU7 & 2925362 & 0.54 & 2930654 & 0.22 & 2943852 & 10.76 & 2943852 & 7620.64 & 7633.53 \\
\hline UU8 & 3959352 & 0.55 & 3959352 & 0.19 & 3969784 & 3.30 & 3970877 & 1822.15 & 1827.64 \\
\hline UW1 & 6036 & 0.58 & 6036 & 0.00 & 6036 & 0.00 & 6036 & 0.72 & 2.83 \\
\hline UW2 & 8468 & 0.66 & 8468 & 0.00 & 8720 & 0.00 & 8720 & 1.45 & 3.48 \\
\hline UW3 & 5888 & 0.50 & 6302 & 0.00 & 6652 & 0.00 & 6652 & 1.20 & 3.18 \\
\hline UW4 & 7748 & 0.52 & 8326 & 0.16 & 8326 & 0.01 & 8326 & 3.24 & 5.31 \\
\hline UW5 & 7780 & 0.64 & 7780 & 0.00 & 7780 & 0.00 & 7780 & 0.74 & 2.76 \\
\hline UW6 & 6548 & 0.67 & 6615 & 0.00 & 6803 & 0.03 & 6803 & 14.68 & 16.77 \\
\hline UW7 & 10464 & 0.65 & 10464 & 0.00 & 10464 & 0.01 & 10464 & 4.42 & 6.47 \\
\hline UW8 & 7692 & 0.66 & 7692 & 0.00 & 7692 & 0.05 & 7692 & 26.79 & 28.92 \\
\hline UW9 & 7038 & 0.72 & 7038 & 0.00 & 7128 & 0.02 & 7128 & 13.87 & 16.02 \\
\hline UW10 & 7461 & 0.67 & 7507 & 0.00 & 7507 & 0.12 & 7507 & 99.79 & 101.96 \\
\hline UW11 & 15747 & 0.70 & 15747 & 0.01 & 16400 & 1.56 & 16400 & 1015.00 & 1018.74 \\
\hline
\end{tabular}

Table 2: Results obtained by the Recursive Partitioning Algorithm for instances of moderate size.

improved the solution given by the previous phase. For example, note that for instances M1 and UU8, the optimal guillotine pattern value coincides with the optimal two-stage pattern value, while the optimal five-block pattern is better and the $L$-Algorithm pattern is even better. Figures 3 and 4 depict the corresponding optimal cutting patterns for instances M1 and UU8, respectively.

As expected, the optimal unconstrained two-stage and guillotine pattern values coincide with the ones reported in the literature $[29,5,39,32,33,30,23,31,1,20,18]$. Moreover, the unconstrained non-guillotine solutions found by the Five-block Algorithm and the $L$-Algorithm improve the guillotine ones in many instances. To the best of our knowledge, there are no studies in the literature reporting unconstrained non-guillotine solutions for these instances. An interesting result in Table 2 is that most of the best solutions of the Recursive Partitioning Approach were found by the Five-block Algorithm, requiring affordable runtimes (only a few seconds).

For instances H and HZ1, the Recursive Partitioning Approach gives an optimality certificate. 


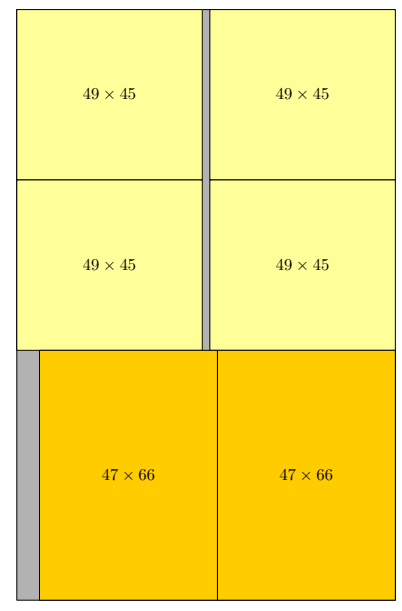

(a)

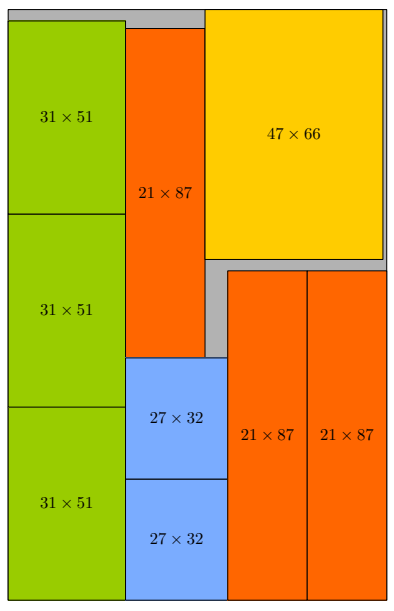

(b)

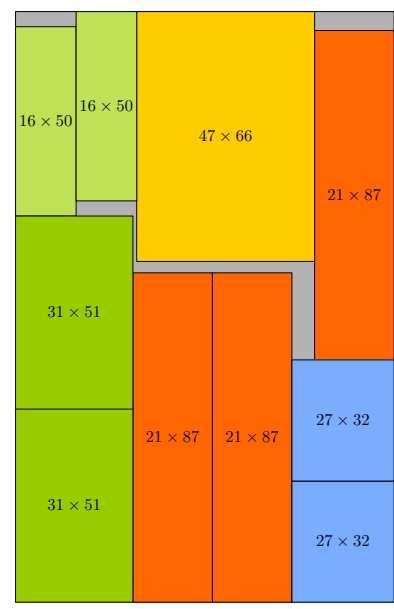

(c)

Figure 3: Graphical representations of solutions found by the Recursive Partitioning Algorithm for instance M1. (a) Optimal guillotine pattern with value 15,024. (b) First-order guillotine pattern with value 15,054 . (c) $L$-Algorithm pattern with value 15,073 .

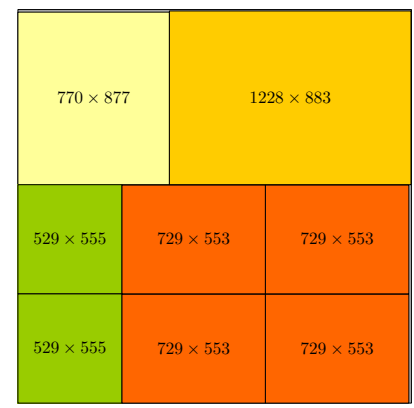

(a)

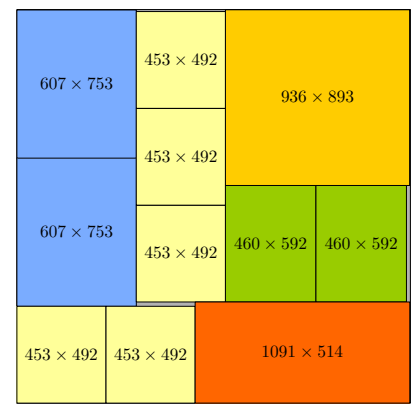

(b)

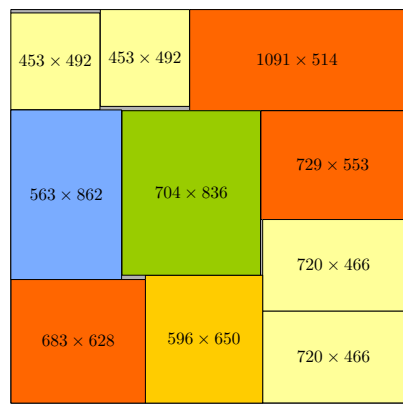

(c)

Figure 4: Graphical representations of solutions found by the Recursive Partitioning Algorithm for instance UU8. (a) Optimal guillotine pattern with value 3,959,352. (b) First-order guillotine pattern with value $3,969,784$. (c) $L$-Algorithm pattern with value $3,970,877$.

For instances HZ2, GCUT1-GCUT3, GCUT5-GCUT7, GCUT9, GCUT10, M1-M5 and MW1MW5, an optimality certificate was obtained solving the LP-relaxation of model (1-3) using the modelling language GAMS 19.8 with the solver CPLEX 7. For all the remaining moderate-sized instances, we were unable to prove the optimality because the CPLEX execution was aborted with a computer memory overflow error.

We also performed additional experiments with other 22 problem instances of the literature $[34,36]$, for which the optimal solutions are known by construction. These unweigthed instances were generated in such a way that there exists at least one optimal cutting pattern 
with zero waste, that is, the value of this cutting pattern coincides with the area of the stock plate. For all instances C1P1-C4P3 in [34] and LCT1-LCT10 in [36], the Recursive Partitioning Approach was able to find an optimal solution. In all cases the optimal pattern is two-stage guillotine, with the exception of instance LCT3 for which the optimal pattern was obtained by the Five-block Algorithm.

\subsection{Large instances}

In a first set of experiments with the large instances, we applied a heuristic version of the Recursive Partitioning Approach, without imposing limits to the search depth (i.e., setting $N=\infty$ ). Sets $S_{L}$ and $S_{W}$ were replaced in the guillotine, the five-block and the $L$-Algorithm phases by sets $\tilde{S}_{L}$ and $\tilde{S}_{W}$ generated by Algorithm 7 with the product threshold parameter $M=40,000,000, M=150,000$ and $M=10,000$, respectively. Therefore, the best guillotine, five-block and non-guillotine solutions obtained by these algorithms may not be optimal.

Table 3 presents the values of the unconstrained two-stage, guillotine, first-order non-guillotine (Five-block Algorithm) and superior-order non-guillotine ( $L$-algorithm) cutting patterns obtained by the heuristic version of the Recursive Partitioning Approach for the large-sized instances. We first note that the two-staged phase of this heuristic version is identical to the one of the exact version of the method, i.e., all two-staged solutions are optimal. Moreover, looking at the quantity $\left|S_{L}\right| \times\left|S_{W}\right|$ of each instance on Table 1, and considering that guillotine cuts of instances with $\left|S_{L}\right| \times\left|S_{W}\right| \leq 40,000,000$ are being solved to optimality, we know that, with the exception of instances LU1-LU4 and LW1-LW4, the guillotine cuts of all the largesized instances are optimal too. Among the optimal guillotine cuts generated by our method, we were able to verify that, for instances GCUT13-GCUT17, U1-U3, W1, W3, UU9-UU11, and APT10-APT29, solutions found by our method are in agreement with previously published solutions $[30,23,31,1,19,20,18]$. For the remaining instances there are no optimal guillotine solutions reported in the literature. Even without this double checking, guillotine solutions for instances U4, W4 and B1-B7 are optimal, but whether or not guillotine solutions for instances LU1-LU4 and LW1-LW4 are optimal remains to be verified. A remark for instances U2 and U3 is in order. Instances U2 and U3 were introduced in [30]. [20] reports their optimal guillotine pattern values as being 20,232,224 and 48,142,840, respectively, mentioning that these optimal values can be obtained by running the algorithm for staged patterns presented in [19] (setting the maximum allowed number of stages to infinity). These values are slightly larger than the ones reported in Table 3, that should be optimal. We implemented and run the method introduced in [19], and the same values reported in Table 3 were found, which suggests that the optimal values for instances U2 and U3 reported in [20] may be incorrect. To reinforce this idea, we note that, as far as we know, there is no publication introducing a method (exact or heuristic) that finds better quality solutions than the ones being reported here, while some publications report equal or lower quality solutions [30, 1, 20].

Regarding Table 3, it is worth remarking that instances LU1-LU4, LW1-LW4 are huge instances and involve tens of thousands of conic combinations. Note that the guillotine solution found for instance B7 is proven to be optimal. Note also that most of the best solutions were obtained by applying only guillotine cuts, but the demanded runtimes are generally much higher than the ones in the moderate-sized instances of Table 2. Exceptions are instances GCUT13, 
W3, W4, UU9 and LU4 for which none of the possible guillotine partitions (neither horizontal nor vertical) provides a lower bound greater than the value of the optimal two-stage pattern. Other exceptions are instances APT21 and APT22 for which the Five-block Algorithm was able to improve the lower bound given by the guillotine partitions. On the other hand, note that the optimal two-stage solutions in the table are reasonably good, if compared to the guillotine solutions, and they are obtained in just a couple of seconds.

\begin{tabular}{|c|c|c|c|c|c|c|c|c|c|}
\hline \multirow{2}{*}{ Instance } & \multicolumn{2}{|c|}{ Two-stage cuts } & \multicolumn{2}{|c|}{ Guillotine cuts } & \multicolumn{2}{|c|}{ Five-block Algorithm } & \multicolumn{2}{|c|}{$L$-Algorithm } & \multirow{2}{*}{$\begin{array}{l}\text { Total } \\
\text { time }\end{array}$} \\
\hline & Solution & Time (sec) & Solution & Time $(\mathrm{sec})$ & Solution & Time $(\mathrm{sec})$ & Solution & Time $(\mathrm{sec})$ & \\
\hline GCUT13 & 8997780 & 0.70 & 8997780 & 21.24 & 8997780 & 10.40 & 8997780 & 0.72 & 36.72 \\
\hline GCUT14 & 12236280 & 0.68 & 12245410 & 206.46 & 12245410 & 37.47 & 12245410 & 46.84 & 298.22 \\
\hline GCUT15 & 12239904 & 0.58 & 12246032 & 225.11 & 12246032 & 29.00 & 12246032 & 876.49 & 1139.46 \\
\hline GCUT16 & 12243100 & 0.57 & 12248836 & 326.06 & 12248836 & 72.24 & 12248836 & 1492.92 & 1901.73 \\
\hline GCUT17 & 12242998 & 0.73 & 12248892 & 430.19 & 12248892 & 28.10 & 12248892 & 1763.37 & 2235.80 \\
\hline U1 & 22167051 & 0.55 & 22370130 & 6.27 & 22370130 & 3.90 & 22370130 & 1.83 & 15.16 \\
\hline $\mathrm{U} 2$ & 20031845 & 0.55 & 20232223 & 0.23 & 20232223 & 0.14 & 20232223 & 0.91 & 3.42 \\
\hline U3 & 47912942 & 0.84 & 48142836 & 14.24 & 48142836 & 0.89 & 48142836 & 1.06 & 23.75 \\
\hline $\mathrm{U} 4$ & 48227224 & 0.76 & 48304289 & 2169.51 & 48304289 & 289.91 & 48304289 & 435.73 & 2917.68 \\
\hline W1 & 161424 & 0.65 & 162867 & 0.16 & 162867 & 0.24 & 162867 & 0.88 & 3.53 \\
\hline W3 & 234108 & 0.70 & 234108 & 0.03 & 234108 & 0.17 & 234108 & 0.82 & 3.31 \\
\hline W4 & 377910 & 0.73 & 377910 & 27.14 & 377910 & 65.67 & 377910 & 158.99 & 261.15 \\
\hline UU9 & 6100692 & 0.62 & 6100692 & 0.19 & 6100692 & 0.03 & 6100692 & 0.88 & 3.97 \\
\hline UU10 & 11929561 & 0.69 & 11955852 & 0.08 & 11955852 & 0.04 & 11955852 & 0.70 & 4.37 \\
\hline UU11 & 13066737 & 0.71 & 13157811 & 217.67 & 13157811 & 988.84 & 13157811 & 244.20 & 1455.46 \\
\hline LU1 & 492259840 & 1.52 & 492278922 & 6792.50 & 492278922 & 154.47 & 492278922 & 1.14 & 7001.03 \\
\hline LU2 & 884318400 & 1.54 & 884341464 & 9620.75 & 884341464 & 88.56 & 884341464 & 0.89 & 9763.91 \\
\hline LU3 & 1035962256 & 2.27 & 1035971468 & 15245.45 & 1035971468 & 116.47 & 1035971468 & 0.92 & 15444.27 \\
\hline LU4 & 1627681752 & 2.35 & 1627681752 & 0.10 & 1627681752 & 154.87 & 1627681752 & 0.90 & 261.50 \\
\hline LW1 & 170590913 & 0.94 & 171245481 & 10313.17 & 171245481 & 145.54 & 171245481 & 15008.65 & 25499.52 \\
\hline LW2 & 324615667 & 0.99 & 325011119 & 9446.71 & 325011119 & 19766.82 & 325011119 & 20104.52 & 49346.43 \\
\hline LW3 & 430291142 & 1.15 & 430706636 & 16192.94 & 430706636 & 94.64 & 430706636 & 19676.79 & 36013.23 \\
\hline LW4 & 565839770 & 1.49 & 566102201 & 11459.56 & 566102201 & 93.23 & 566102201 & 22218.03 & 33833.78 \\
\hline APT10 & 3585612 & 0.56 & 3589703 & 56.52 & 3589703 & 190.66 & 3589703 & 1727.17 & 1978.77 \\
\hline APT11 & 4175414 & 0.54 & 4188915 & 81.59 & 4188915 & 50.09 & 4188915 & 2748.75 & 2885.51 \\
\hline APT12 & 5148302 & 0.54 & 5156065 & 21.16 & 5156065 & 270.93 & 5156065 & 174.00 & 469.79 \\
\hline APT13 & 3493072 & 0.73 & 3498302 & 56.41 & 3498302 & 60.40 & 3498302 & 0.87 & 122.27 \\
\hline APT14 & 4458052 & 0.55 & 4463550 & 51.57 & 4463550 & 89.45 & 4463550 & 1082.57 & 1227.69 \\
\hline APT15 & 6028426 & 0.72 & 6047188 & 70.68 & 6047188 & 81.15 & 6047188 & 669.11 & 826.36 \\
\hline APT16 & 7533987 & 0.70 & 7566719 & 98.33 & 7566719 & 62.66 & 7566719 & 893.13 & 1060.57 \\
\hline APT17 & 4529371 & 0.56 & 4535302 & 88.67 & 4535302 & 70.07 & 4535302 & 1540.74 & 1704.73 \\
\hline APT18 & 5807988 & 0.66 & 5825956 & 39.50 & 5825956 & 129.76 & 5825956 & 215.25 & 388.37 \\
\hline AРT19 & 6811338 & 0.51 & 6826674 & 48.88 & 6826674 & 28.93 & 6826674 & 337.51 & 419.70 \\
\hline APT20 & 5490828 & 0.54 & 5545818 & 112.36 & 5545818 & 8114.96 & 5545818 & 1039.89 & 9272.29 \\
\hline АРТ 21 & 3479634 & 0.52 & 3484406 & 25.14 & 3491545 & 247.33 & 3491545 & 358.85 & 635.07 \\
\hline АРТ 22 & 4111542 & 0.55 & 4145317 & 70.56 & 4153426 & 5139.29 & 4153426 & 1224.32 & 6438.58 \\
\hline АРТ 23 & 3494778 & 0.53 & 3546535 & 25.99 & 3546535 & 1067.21 & 3546535 & 559.38 & 1656.34 \\
\hline АРT24 & 3898694 & 0.69 & 3948037 & 73.38 & 3948037 & 12497.31 & 3948037 & 1639.73 & 14214.76 \\
\hline APT 25 & 3485589 & 0.67 & 3507615 & 36.38 & 3507615 & 2026.71 & 3507615 & 612.73 & 2679.23 \\
\hline АРТ 26 & 2639964 & 0.64 & 2683689 & 33.17 & 2683689 & 2290.99 & 2683689 & 985.56 & 3313.24 \\
\hline APT 27 & 2382270 & 0.66 & 2438174 & 19.20 & 2438174 & 1283.44 & 2438174 & 412.72 & 1718.58 \\
\hline АРT 28 & 3969356 & 0.52 & 4065011 & 79.46 & 4065011 & 7946.36 & 4065011 & 2079.59 & 10109.82 \\
\hline АРТ 29 & 3619071 & 0.50 & 3652858 & 63.10 & 3652858 & 5255.54 & 3652858 & 1276.47 & 6599.48 \\
\hline B1 & 7990710 & 0.68 & 7993031 & 118.17 & 7993031 & 25.13 & 7993031 & 337.64 & 485.55 \\
\hline B2 & 7984124 & 0.74 & 7993849 & 108.62 & 7993849 & 149.35 & 7993849 & 395.13 & 657.75 \\
\hline B3 & 7966146 & 0.67 & 7991615 & 129.17 & 7991615 & 35.04 & 7991615 & 406.55 & 575.43 \\
\hline B4 & 7979928 & 0.54 & 7989673 & 135.13 & 7989673 & 1316.49 & 7989673 & 492.63 & 1948.81 \\
\hline B5 & 7978160 & 0.55 & 7994752 & 101.15 & 7994752 & 30.28 & 7994752 & 319.74 & 455.67 \\
\hline B6 & 7989831 & 0.66 & 7992075 & 107.35 & 7992075 & 458.75 & 7992075 & 413.29 & 983.88 \\
\hline B7 & 7999488 & 0.83 & 8000000 & 47.09 & - & - & - & - & 66.58 \\
\hline
\end{tabular}

Table 3: Results obtained by a heuristic version of the Recursive Partitioning Algorithm for the large instances. The sets of conic combinations were limited in the guillotine, five-block and $L$-Algorithm phases of the method.

In a second set of experiments, we applied another heuristic version of the Recursive Partitioning Approach, using the complete conic combinations sets in all the phases of the method, fixing the search depth limit parameter $N=\infty$ in the guillotine phase and $N=1$ in the fiveblock phase, and skipping the $L$-Algorithm phase of the method. Table 4 presents the values 
of the unconstrained two-stage, guillotine and first-order non-guillotine (Five-block Algorithm) cuts obtained by this heuristic version of the Recursive Partitioning Approach for some of the large-sized instances. The unconstrained two-stage and guillotine pattern values coincide with the ones of Table 3. Note, however, that the Five-block Algorithm was able to find improved non-guillotine solutions for most of these examples (if compared to Table 3), at the expenses of much higher running times. In particular, since the upper bound on the optimal value for instance GCUT14-GCUT17 provided by the algorithm is 12,250,000, we note that the five-block solution found for instance GCUT17 is proven to be optimal. Besides that, note that, for instance APT22, the heuristic version of the Five-block Algorithm with $N=1$ and using the complete set of conic combinations found a better solution than the previous heuristic version with $N=\infty$ and a shrunk set of conic combinations (see Table 3 ).

\begin{tabular}{|l|c|c|c|c|c|c|c|}
\hline \multirow{2}{*}{ Instance } & \multicolumn{2}{|c}{ Two-stage } & \multicolumn{2}{c|}{ Guillotine cuts } & \multicolumn{2}{c|}{ Five-block } & Total \\
\cline { 2 - 8 } & Solution & Time (sec) & Solution & Time (sec) & Solution & Time (sec) & time \\
\hline \hline GCUT14 & 12236280 & 0.70 & $\mathbf{1 2 2 4 5 4 1 0}$ & 205.18 & $\mathbf{1 2 2 4 9 2 0 0}$ & 150212.43 & 150422.13 \\
GCUT15 & 12239904 & 0.60 & $\mathbf{1 2 2 4 6 0 3 2}$ & 224.15 & $\mathbf{1 2 2 4 9 2 0 0}$ & 172000.08 & 172229.49 \\
GCUT16 & 12243100 & 0.70 & $\mathbf{1 2 2 4 8 8 3 6}$ & 312.87 & $\mathbf{1 2 2 4 9 2 0 0}$ & 227771.90 & 228091.34 \\
GCUT17 & 12242998 & 0.61 & $\mathbf{1 2 2 4 8 8 9 2}$ & 408.31 & $\mathbf{1 2 2 5 0 0 0 0}$ & 245000.98 & 245417.90 \\
\hline APT10 & 3585612 & 0.68 & $\mathbf{3 5 8 9 7 0 3}$ & 54.98 & $\mathbf{3 5 8 9 8 3 3}$ & 22263.79 & 22319.45 \\
APT11 & 4175414 & 0.56 & $\mathbf{4 1 8 8 9 1 5}$ & 81.36 & $\mathbf{4 1 8 9 8 6 0}$ & 33014.50 & 33099.53 \\
APT12 & 5148302 & 0.72 & $\mathbf{5 1 5 6 0 6 5}$ & 20.22 & $\mathbf{5 1 5 6 6 1 8}$ & 9256.83 & 9279.67 \\
APT13 & 3493072 & 0.71 & $\mathbf{3 4 9 8 3 0 2}$ & 55.75 & 3498302 & 22523.98 & 22583.18 \\
APT14 & 4458052 & 0.54 & $\mathbf{4 4 6 3 5 5 0}$ & 50.40 & 4463550 & 20573.89 & 20627.23 \\
APT15 & 6028426 & 0.73 & $\mathbf{6 0 4 7 1 8 8}$ & 70.08 & $\mathbf{6 0 5 2 2 7 7}$ & 36959.17 & 37032.87 \\
APT16 & 7533987 & 0.52 & $\mathbf{7 5 6 6 7 1 9}$ & 96.11 & $\mathbf{7 5 7 1 5 9 0}$ & 51597.46 & 51697.39 \\
APT17 & 4529371 & 0.57 & $\mathbf{4 5 3 5 3 0 2}$ & 87.79 & $\mathbf{4 5 3 6 3 7 2}$ & 33850.66 & 33942.20 \\
APT18 & 5807988 & 0.72 & $\mathbf{5 8 2 5 9 5 6}$ & 36.83 & $\mathbf{5 8 2 9 1 7 2}$ & 15196.78 & 15236.43 \\
APT19 & 6811338 & 0.56 & $\mathbf{6 8 2 6 6 7 4}$ & 48.43 & 6826674 & 27025.49 & 27076.86 \\
APT20 & 5490828 & 0.70 & $\mathbf{5 5 4 5 8 1 8}$ & 111.39 & $\mathbf{5 5 4 8 5 0 5}$ & 59086.20 & 59201.01 \\
APT21 & 3479634 & 0.67 & $\mathbf{3 4 8 4 4 0 6}$ & 24.58 & $\mathbf{3 4 9 1 5 4 5}$ & 12537.07 & 12564.35 \\
APT22 & 411542 & 0.56 & $\mathbf{4 1 4 5 3 1 7}$ & 69.35 & $\mathbf{4 1 6 1 3 8 4}$ & 32738.22 & 32810.68 \\
APT23 & 3494778 & 0.71 & $\mathbf{3 5 4 6 5 3 5}$ & 25.49 & $\mathbf{3 5 5 9 0 2 8}$ & 13606.86 & 13635.08 \\
APT24 & 3898694 & 0.68 & $\mathbf{3 9 4 8 0 3 7}$ & 73.03 & $\mathbf{3 9 5 6 4 9 8}$ & 36299.42 & 36375.59 \\
APT25 & 3485589 & 0.70 & $\mathbf{3 5 0 7 6 1 5}$ & 34.62 & 3507615 & 14851.38 & 14888.59 \\
APT26 & 2639964 & 0.50 & $\mathbf{2 6 8 3 6 8 9}$ & 32.43 & 2683689 & 13092.85 & 13127.84 \\
APT27 & 2382270 & 0.56 & $\mathbf{2 4 3 8 1 7 4}$ & 18.42 & $\mathbf{2 4 4 3 6 9 6}$ & 6770.92 & 6791.78 \\
APT28 & 3969356 & 0.56 & $\mathbf{4 0 6 5 0 1 1}$ & 79.07 & 4065011 & 39197.62 & 39279.83 \\
APT29 & 3619071 & 0.70 & $\mathbf{3 6 5 2 8 5 8}$ & 61.30 & 3652858 & 28104.65 & 28169.23 \\
\hline
\end{tabular}

Table 4: Results obtained by a heuristic version of the Recursive Partitioning Approach for instances GCUT14-GCUT17 and APT10-APT29. The search depth limit was set to $N=1$ in the five-block phase and the complete conic combinations sets were used in all the phases of the method.

In addition to the experiments described above, we arbitrarily decided to solve the two large instances UU9 and UU10 with the non-heuristic version of the Recursive Partitioning Approach. While for instance UU9 the best solution obtained corresponds to a two-stage pattern with value $6,100,692$, for instance UU10, each phase of the method improved the solution found by the previous phase. Figure 5 illustrates the corresponding cutting patterns obtained by each phase for instance UU10. The total runtimes for solving instances UU9 and UU10 were 4,321.59 and $8,908.24$ seconds, respectively.

A final remark on the preprocessing scheme of the input of the Recursive Partitioning Algorithm is in order. The preprocessing scheme for eliminating dominated items, described in the last paragraph of Subsection 3.3, applies to weighted instances only (35 over a total of 95). Table 1 showed the number $m$ of original items and the number $m_{\mathrm{r}}$ of remaining items after applying the preprocessing scheme to each instance. A reduction in the number of items may 


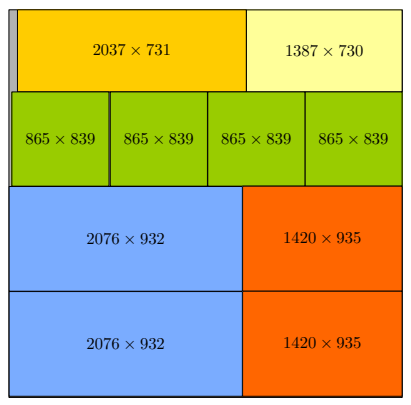

(a)

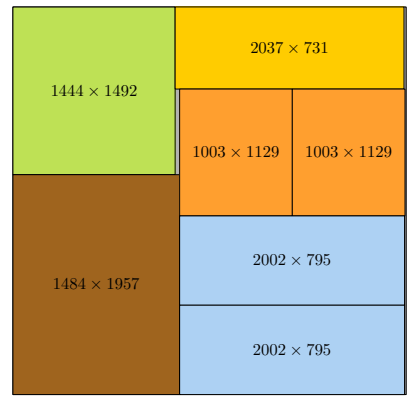

(c)

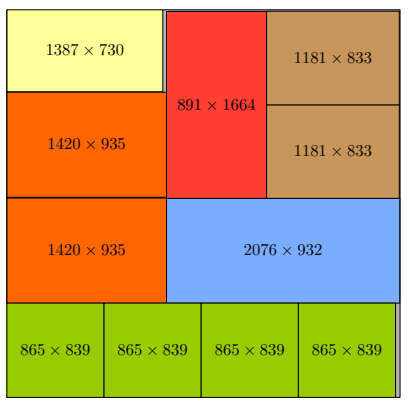

(b)

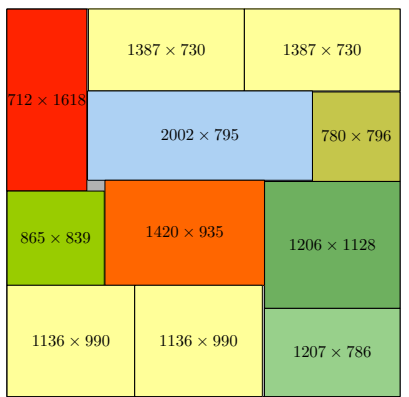

(d)

Figure 5: Graphical representations of solutions found by the Recursive Partitioning Algorithm for instance UU10. (a) Optimal two-stage pattern with value 11,929,561. (b) Optimal guillotine pattern with value 11,955,852. (c) First-order guillotine pattern with value 11,995,637. (d) $L$-Algorithm pattern with value 12,001,291.

imply in a reduction on the size of the conic combinations and raster points sets. Considering the 35 weighted instances, the average reduction in the product $\left|S_{L}\right|\left|S_{W}\right|$ was $42.80 \%$ while the average reduction in the product $\left|R_{L}\right|\left|R_{W}\right|$ was $46.02 \%$. Extreme cases were instances HZ2 and MW1, for which there was no reduction at all, and instances UW5 and W3. Instance UW5 had a reduction of $97.60 \%$ in the product $\left|S_{L}\right|\left|S_{W}\right|$, while instance W3 had a reduction of $99.16 \%$ in the product $\left|R_{L}\right|\left|R_{W}\right|$. We illustrate the running time reduction in instances UW6, UW8 and UW9, for which the reductions in $\left|S_{L}\right|\left|S_{W}\right|$ and $\left|R_{L}\right|\left|R_{W}\right|$ were, $78.67 \%$ and $84.57 \%, 82.76 \%$ and $90.66 \%$, and $90.13 \%$ and $78.21 \%$, respectively. The running time for solving instance UW6 without preprocessing was $1,837.47$ seconds, while this time went down to 16.77 seconds when using the preprocessing strategy. For instances UW8 and UW9, the running times without preprocessing were 10,639.46 and 802.19 seconds, respectively, and, 28.92 and 16.02 seconds with preprocessing, respectively. 


\section{Concluding remarks}

While a large number of studies in the literature have considered staged and non-staged two-dimensional guillotine cutting problems, much less studies have considered two-dimensional non-guillotine cutting problems (constrained and unconstrained), and only a few of them have proposed exact methods to generate non-guillotine patterns. Moreover, most of the approaches (exact and heuristic) for non-guillotine cutting (or packing) were developed for the constrained problem, which can be more interesting for certain practical applications with relatively low demands of the ordered items. However, part of these methods may not perform well when solving the unconstrained problem. On the other hand, the unconstrained problem is particularly interesting for cutting stock applications with large-scale production and weakly heterogeneous items, in which the problem plays the role of a column generation procedure.

This study presented a Recursive Partitioning Approach to generate unconstrained twodimensional non-guillotine cutting (or packing) patterns. The approach was able to find the optimal solution of a large number of moderate-sized instances known in the literature and we were unable to find a counterexample for which the approach fails to find a known optimal solution. To cope with large instances, we combined the approach with simple heuristics to reduce its computational efforts. For moderate-sized instances, both the five-block and the $L$ Algorithm phases of the approach seem to be promising alternatives for obtaining reasonably good or optimal non-guillotine solutions under affordable computer runtimes, whereas for larger instances, the guillotine or the five-block phase may be preferable, depending on the definition of an acceptable time limit. An interesting perspective for future research is to extend the Recursive Partitioning Approach to deal with constrained two-dimensional non-guillotine cutting.

\section{References}

[1] R. Alvarez-Valdés, A. Parajón, and J. M. Tamarit. A tabu search algorithm for large-scale guillotine (un)constrained two-dimensional cutting problems. Computers and Operations Research, 29:925-947, 2002.

[2] R. Alvarez-Valdés, F. Parreño, and J. M. Tamarit. A tabu search algorithm for a twodimensional non-guillotine cutting problem. European Journal of Operational Research, 183:1167-1182, 2007.

[3] M. Arenales and R. Morabito. An and/or-graph approach to the solution of two-dimensional non-guillotine cutting problems. European Journal of Operational Research, 84:599-617, 1995.

[4] R. Baldacci and M. A. Boschetti. A cutting-plane approach for the two-dimensional orthogonal non-guillotine cutting problem. European Journal of Operational Research, 183:11361149, 2007.

[5] J. E. Beasley. Algorithms for unconstrained two-dimensional guillotine cutting. Journal of the Operational Research Society, 36:297-306, 1985. 
[6] J. E. Beasley. An exact two-dimensional non-guillotine cutting tree-search procedure. $O p$ erations Research, 33:49-64, 1985.

[7] J. E. Beasley. A population heuristic for constrained two-dimensional non-guillotine cutting. European Journal of Operational Research, 156:601-627, 2004.

[8] E. G. Birgin and R. D. Lobato. Orthogonal packing of identical rectangles within isotropic convex regions. Computers ES Industrial Engineering. DOI: 10.1016/j.cie.2010.07.004.

[9] E. G. Birgin, R. D. Lobato, and R. Morabito. An effective recursive partitioning approach for the packing of identical rectangles in a rectangle. Journal of the Operational Research Society, 61:306-320, 2010.

[10] E. G. Birgin, J. M. Martínez, W. F. Mascarenhas, and D. P. Ronconi. Method of sentinels for packing items within arbitrary convex regions. Journal of the Operational Research Society, 57:735-746, 2006.

[11] E. G. Birgin, J. M. Martínez, F. H. Nishihara, and D. P. Ronconi. Orthogonal packing of rectangular items within arbitrary convex regions by nonlinear optimization. Computers and Operations Research, 33:3535-3548, 2006.

[12] E. G. Birgin, R. Morabito, and F. H. Nishihara. A note on an $L$-approach for solving the manufacturer's pallet loading problem. Journal of the Operational Research Society, 56:1448-1451, 2005.

[13] A. Bortfeldt and T. Winter. A genetic algorithm for the two-dimensional knapsack problem with rectangular pieces. International Transactions in Operational Research, 16:685-713, 2009.

[14] M. A. Boschetti, A. Mingozzi, and E. Hadjiconstantinou. New upper bounds for the twodimensional orthogonal non-guillotine cutting stock problem. IMA Journal of Management Mathematics, 13:95-119, 2002.

[15] A. Caprara and M. Monaci. On the two-dimensional knapsack problem. Operations Research Letters, 32:5-14, 2004.

[16] C. S. Chen, S. M. Lee, and Q. S. Shen. An analytical model for the container loading problem. European Journal of Operational Research, 80:68-76, 1995.

[17] D. Chen and W. Huang. A new heuristic algorithm for constrained rectangle-packing problem. Asia-Pacific Journal of Operational Research, 24:463-478, 2007.

[18] G. F. Cintra, F. K. Miyazawa, Y. Wakabayashi, and E. C. Xavier. Algorithms for twodimensional cutting stock and strip packing problems using dynamic programming and column generation. European Journal of Operational Research, 191:61-85, 2008.

[19] Y. Cui, Z. Wang, and J. Li. Exact and heuristic algorithms for staged cutting problems. Proceedings of the Institution of Mechanical Engineers, Part B: Journal of Engineering Manufacture, 219:201-207, 2005. 
[20] Y. Cui and X. Zhang. Two-stage general block patterns for the two-dimensional cutting problem. Computers and Operations Research, 34:2882-2893, 2007.

[21] H. Dyckhoff. A typology of cutting and packing problems. European Journal of Operational Research, 44:145-159, 1990.

[22] J. Egeblad and D. Pisinger. Heuristic approaches for the two- and three-dimensional knapsack packing problem. Computers and Operations Research, 36:1026-1049, 2009.

[23] D. Fayard, M. Hifi, and V. Zissimopoulos. An efficient approach for large-scale twodimensional guillotine cutting stock problems. Journal of the Operational Research Society, 49:1270-1277, 1998.

[24] S. P. Fekete, J. Schepers, and J. C. van der Veen. An exact algorithm for higher-dimensional orthogonal packing. Operations Research, 55:569-587, 2007.

[25] Y.-G. G and M.-K. Kang. A new upper bound for unconstrained two-dimensional cutting and packing. Journal of the Operational Research Society, 53:587-591, 2002.

[26] P. C. Gilmore and R. E. Gomory. Multistage cutting stock problems of two and more dimensions. Operations Research, 13:94-120, 1965.

[27] J. F. Gonçalves. A hybrid genetic algorithm-heuristic for a two-dimensional orthogonal packing problem. European Journal of Operational Research, 183:1212-1229, 2007.

[28] E. Hadjiconstantinou and N. Christofides. An exact algorithm for general, orthogonal, 2-dimensional knapsack-problems. European Journal of Operational Research, 83:39-56, 1995.

[29] J. C. Herz. Recursive computational procedure for two-dimensional stock cutting. IBM Journal of Research and Development, 16:462-469, 1972.

[30] M. Hifi. The DH/KD algorithm: a hybrid approach for unconstrained two-dimensional cutting problems. European Journal of Operational Research, 97:41-52, 1997.

[31] M. Hifi. Exact algorithms for large-scale unconstrained two and three staged cutting problems. Computational Optimization and Applications, 18:63-88, 2001.

[32] M. Hifi and V. Zissimopoulos. A recursive exact algorithm for weighted two-dimensional cutting. European Journal of Operational Research, 91:553-564, 1996.

[33] M. Hifi and V. Zissimopoulos. Une amélioration de l'algorithme récursif de Herz pour le problème de découpe à deux dimensions. RAIRO, 30:111-125, 1996.

[34] E. Hopper and B. C. H. Turton. An empirical investigation of meta-heuristic and heuristic algorithms for a 2D packing problem. European Journal of Operational Research, 128:34-57, 2001.

[35] W. Huang and D. Chen. An efficient heuristic algorithm for rectangle-packing problem. Simulation Modelling Practice and Theory, 15:1356-1365, 2007. 
[36] T. W. Leung, C. K. Chan, and M. D. Troutt. Application of a mixed simulated annealinggenetic algorithm heuristic for the two-dimensional orthogonal packing problem. European Journal of Operational Research, 145:530-542, 2003.

[37] L. Lins, S. Lins, and R. Morabito. An $L$-approach for packing $(l, w)$-rectangles into rectangular and $L$-shaped pieces. Journal of the Operational Research Society, 54:777-789, 2003.

[38] W. F. Mascarenhas and E. G. Birgin. Using sentinels to detect intersections of convex and nonconvex polygons. Computational \& Applied Mathematics, 29:247-267, 2010.

[39] R. Morabito, M. Arenales, and V. F. Arcaro. An and-or-graph approach for two-dimensional cutting problems. European Journal of Operational Research, 58:263-271, 1992.

[40] R. Morabito and S. Morales. A simple and effective recursive procedure for the manufacturer's pallet loading problem. Journal of the Operational Research Society, 49:819-828, 1998.

[41] R. Morabito and S. Morales. Erratum to 'A simple and effective recursive procedure for the manufacturer's pallet loading problem'. Journal of the Operational Research Society, 50:876-876, 1999.

[42] M. Padberg. Packing small boxes into a big box. Mathematical Methods of Operations Research, 52:1-21, 2000.

[43] G. Scheithauer. LP-based bounds for the container and multi-container loading problem. International Transactions in Operational Research, 6:199-213, 1999.

[44] G. Scheithauer and J. Terno. Modeling of packing problems. Optimization, 28:63-84, 1993.

[45] G. Scheithauer and J. Terno. The G4-heuristic for the pallet loading problem. Journal of the Operational Research Society, 47:511-522, 1996.

[46] R. D. Tsai, E.E. Malstrom, and W. Kuo. 3-dimensional palletization of mixed box sizes. IIE Transactions, 25:64-75, 1993.

[47] G. Wäescher, H. Haußner, and H. Schumann. An improved typology of cutting and packing problems. European Journal of Operational Research, 183:1109-1130, 2007.

[48] L. Wei, D. Zhang, and Q. Chen. A least wasted first heuristic algorithm for the rectangular packing problem. Computers and Operations Research, 36:1608-1614, 2009.

[49] http://www.ime.usp.br/ egbirgin/packing/. 